\title{
Theorie des pn-Kontaktes zwischen Halbleitern mit verschiedenen Kristallgittern
}

\author{
Von U. Dolega \\ Standard Elektrik Lorenz AG, Stuttgart-Zuffenhausen \\ (Z. Naturforschg. 18 a, 653-666 [1963] ; eingegangen am 13. Dezember 1962)
}

\begin{abstract}
On the basis of the Sснотткy space charge theory of semiconductor rectifiers a theory for pnheterojunctions is given. The presence of a thin layer with an extremely small carrier lifetime at the interface between the two semiconductors is considered to be typical for pn-heterojunctions. The pn-heterojunction is found to correspond to two metal-semiconductor contacts in series, the boundary concentrations of current carriers, however, not being independent upon the voltage applied. The d.c. properties are mainly determined by the ratio of the densities of imperfections in the two semiconductors. The straight-line portion of the logarithmic forward characteristic has a slope between $e / k T$ and $e / 2 k T$, depending on the value of this ratio. The reverse current rises exponentially with voltage; the reverse resistance has a maximum.
\end{abstract}

\section{Einführung}

Die vorliegende Arbeit ist das Ergebnis von Bemühungen um eine verbesserte Theorie des Selengleichrichters, dessen prinzipielle Wirkungsweise vor 23 Jahren durch die Scнотткуsche Raumladungstheorie der Halbleitergleichrichter ${ }^{1,2}$ geklärt wurde. Das Entscheidende dieser Theorie war die Erkenntnis, daß in der Randschicht eines Halbleiters besondere Verhältnisse hinsichtlich der Konzentration der Stromträger und des Potentialverlaufes herrschen, und daß diese Verhältnisse von einer über die Randschicht von außen angelegten Spannung abhängen. Sснотткy und Spenke bauten diese Theorie besonders als Theorie des Metall-Halbleiter-Kontaktes ${ }^{3-5}$ aus und wandten sie auf den Selengleichrichter an. Dabei ergab sich Übereinstimmung in folgenden Punkten:

1. Der Gleichrichtungssinn wurde in Abhängigkeit vom Leitungscharakter des Halbleiters richtig wiedergegeben.

2. Die berechnete Gleichstromkennlinie stimmte für nicht zu hohe Spannungen in Fluß- und Sperrrichtung qualitativ mit den Experimenten überein.

3. Die aus der Theorie folgende Beziehung für die Spannungsabhängigkeit der Kapazität der Raumladungsschicht gestattete es, aus entsprechenden Mes-

1 W. Sсноттку, Z. Phys. 113, 367 [1939].

2 W. Schotrky u. E. Spenke, Wiss. Veröff. Siemens-Werke 18, Heft 3 [1939].

3 E. Spenke, Wiss. Veröff. Siemens-Werke 20, 40 [1941].

4 W. Sсноттку, Z. Phys. 118, 539 [1942].

5 E. SPEnke, Z. Phys. 126, 67 [1949].

${ }^{6}$ H. Schweickert, Verh. dtsch. phys. Ges. 3, 99 [1939]. sungen die Dicke der Raumladungsschicht und die Störstellendichte des Halbleiters zu berechnen. Die so ermittelten Werte stimmten gut mit solchen überein, die durch andere Meßmethoden gewonnen waren.

Bestehen blieben jedoch drei Unterschiede zwischen der Theorie und Messungen an Selengleichrichtern. Einmal zeigten die Experimente für gröBere Spannungen in Sperrichtung einen wesentlich steileren Anstieg des Stromes als die Theorie. Zum anderen bestätigte sich die aus der Theorie folgende Abhängigkeit des Nullwiderstandes eines MetallHalbleiter-Kontaktes von der Art des Metalls über dessen Elektronen-Austrittsarbeit nur qualitativ ${ }^{6,7}$. Schließlich ergeben Messungen an Selengleichrichtern, daß der Strom in Flußrichtung für $U>k T / e$ mit $\exp \{e U / a k T\}$ ansteigt, wobei $a$ zwischen 1 und 3 liegt und vorzugsweise etwa den Wert 2 hat, während die Theorie $a=1$ ergibt.

Nun konnten in den Jahren 1949/50 unabhängig voneinander Tomura ${ }^{8}$, Poganski ${ }^{9}$ sowie Yamaguchi und Katayama ${ }^{10}$ nachweisen, daß sich bei der technischen Herstellung von Selengleichrichtern zwischen dem Cd-haltigen Deckelektrodenmetall und dem Selen eine Zwischenschicht aus CdSe bildet. Während Tomura ${ }^{8,11}$ die CdSe-Schicht als Isolator auffaßte und auf sein Modell die Theorie von Motт ${ }^{12}$ an-

7 S. Poganski, Z. Phys. 134, 469 [1953].

8 M. Tomura, Bull. Chem. Soc., Japan 22, 82 [1949].

9 S. Poganski, Diss. Technische Universität Berlin 1950.

10 J. Yamaguchi u. S. Katayama, J. Phys. Soc., Japan 5, 385 [1950].

11 M. Tomura, J. Phys. Soc., Japan 5, 222 [1950].

12 N. F. Мотт, Proc. Roy. Soc., Lond. A 171, 27 [1939]. 
wendete, war es Poganski ${ }^{9}$, der als erster den Schluß zog, daß der eigentliche Sperrmechanismus sich beim Selengleichrichter nicht an der Grenze Metall/Halbleiter, sondern an der Grenze CdSe/Se abspielt, wobei er das Cadmiumselenid als n-Leiter ansah. Die Richtigkeit dieser Vorstellung wurde in den folgenden Jahren durch weitere Untersuchungen von PoGANSKI $^{7,13}$, Hoffmann und Rose ${ }^{14}$ sowie Yama${ }_{\text {GUCHI }}{ }^{15}$ nachgewiesen. Weitere Arbeiten über Selengleichrichter mit Selenidschichten zwischen dem Deckelektrodenmetall und dem Selen von Strosche ${ }^{16,17}$ und Moriguchi ${ }^{18}$, zeigten ebenfalls, welch großen Einfluß diese Zwischenschichten auf das Gleichrichterverhalten haben.

Nachdem somit feststand, daß die wirksame Sperrschicht des Selengleichrichters nicht durch einen Metall-Halbleiter-Kontakt, sondern durch den Kontakt zwischen n-leitendem $\mathrm{CdSe}$ und p-leitendem Selen gebildet wird, konnte man gar nicht mehr erwarten, daß die Sснотткуsche Raumladungstheorie des Metall-Halbleiter-Kontaktes das Verhalten des Selengleichrichters im einzelnen richtig beschreiben würde. Eine verbesserte Theorie des Selengleichrichters mußte vielmehr von einer Anordnung

$$
\text { n-Leiter | p-Leiter }
$$

ausgehen, wobei die beiden Halbleiter chemisch verschieden sind und verschiedene Kristallgitter besitzen. Eine solche Anordnung wollen wir "pnKontakt“ ("pn-heterojunction“) nennen im Gegensatz zu einem „pn-Übergang“ („pn-junction“), bei dem beide Halbleiter das gleiche Kristallgitter besitzen.

Eine Theorie des pn-Kontaktes hat Gubanow ${ }^{19}$ in der ersten einer Reihe von Arbeiten veröffentlicht, welche von Poganski ${ }^{20}$ referiert wurden. Gubanow benutzt ein Näherungsverfahren zur Lösung der dem pn-Kontakt zugrunde liegenden Differentialgleichungen, indem er den für den stromlosen Zustand geltenden Zusammenhang zwischen der Konzentration der Stromträger ( $\mathrm{n}$ im Falle eines n-Leiters) und dem Potentialverlauf $V(x)$,

$$
n(x)=n(V=0) \exp \{e V(x) / k T\},
$$

den man gewöhnlich als Boltzmann-Verteilung bezeichnet, auch für den stromdurchflossenen pn-Kon-

13 S. Poganski, Z. Elektrochem. 56, 193 [1952].

14 A. Hoffmann u. F. Rose, Z. Phys. 136, 152 [1953].

15 J. Yamaguchi et al., J. Phys. Soc., Japan 10, 233 [1955].

16 H. Strosche, Z. Phys. 140, 409 [1955].

17 H. Strosche, Z. Phys. 145, 597 [1956]. takt gelten läßt. Da die Boltzmann-Verteilung aber gerade gegenseitige Kompensation von Feld- und Diffusionsstrom bedeutet, schränkt dieses Näherungsverfahren den Gültigkeitsbereich der Lösungen auf den Teil der Strom-Spannungs-Kennlinie ein, in dem Feld- und Diffusionsstrom wenigstens annähernd gleich sind. Die Lösungen gelten daher, wie auch Gubanow selbst angibt, in Sperrichtung nur bis zu Spannungen von etwa 1 Volt.

Da aber gerade ein besseres theoretisches Verständnis des steilen Anstieges des Sperrstromes eines Selengleichrichters für Spannungen über $10 \mathrm{~V}$ von besonderem Interesse ist, hat der Verfasser versucht, auf der Grundlage der Sснотткуschen Raumladungstheorie eine Theorie des pn-Kontaktes zu entwickeln, welche insbesondere auch für hohe Spannungen in Sperrichtung Gültigkeit besitzt.

\section{Grundeigenschaft des pn-Kontaktes: die Grenzschicht mit verschwindender Lebens- dauer der Stromträger}

Wir betrachten einen p- und einen n-Leiter, die in innigem Kontakt miteinander stehen mögen. Für die Dichten der Stromträger an der Grenze der beiden Halbleiter bestehen, zunächst im thermischen Gleichgewicht, gewisse Randbedingungen. Im allgemeinen werden hierdurch an dieser Grenze andere Konzentrationen der Stromträger herrschen als im Inneren der Halbleiter; es werden Anreicherungs- oder Verarmungsrandschichten entstehen. Wir wollen hier nur den Fall von Verarmungsrandschichten näher betrachten, da nur dieser zu ausgeprägten Gleichrichtereigenschaften führt. Dann bilden sich, von der Grenze der beiden Halbleiter ausgehend, im pLeiter eine negative und im n-Leiter eine positive Raumladungsschicht aus.

Fließt durch eine derartige ebene Anordnung ein Strom, so wird dieser im Inneren des n-Leiters vorwiegend ein Elektronenstrom, im Inneren des p-Leiters vorwiegend ein Defektelektronenstrom sein. Durch Rekombinations- und Generationsvorgänge werden diese beiden Ströme in mehr oder weniger ausgedehnten Bereichen beiderseits der Grenze der beiden Halbleiter ineinander überführt (Abb. 1 a) .

18 Y. Moriguchi, J. Phys. Soc., Japan 14, 152 [1959].

19 A. I. Gubanow, J. Tech. Phys., U.S.S.R. 20, 1287 [1950].

20 S. Poganski, Halbleiterprobleme I, Herausg.: W. Sсноттку, Verlag Vieweg, Braunschweig 1954. 
Handelt es sich um einen pn-Übergang, bei dem sich das Kristallgitter an der Grenze der beiden Halbleiter ungestört fortsetzt, so wird an dieser Grenze die Dichte der Rekombinationszentren nicht größer als im Inneren der Halbleiter sein. Sind weiter die Diffusionslängen der Minoritätsträger im pund n-Leiter größer als die Ausdehnungen der Raumladungsschichten, so kann man die Rekombination innerhalb der Raumladungsschichten vernachlässigen (Abb. $1 \mathrm{~b}$ ). Dies ist der Ausgangspunkt der Theorie des pn-Überganges von SHоскLеу ${ }^{21}$.
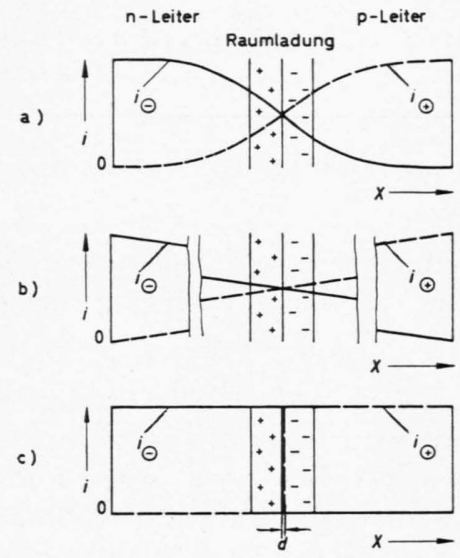

Abb. 1. Stromübernahme an der Grenze zwischen einem n- und einem p-Leiter. a) allgemeiner Fall; Rekombination in und außerhalb der Raumladungsschicht; b) „keine“ Rekombination innerhalb der Raumladungsschicht; Theorie des pn-Uberganges von SHockLEY; c) hohe Rekombination innerhalb einer sehr dünnen Schicht; Kennzeichen des pn-Kontaktes.

$\mathrm{i} \ominus$ : Elektronenstrom, $\mathrm{i} \oplus$ : Defektelektronenstrom.

Handelt es sich jedoch um einen pn-Kontakt, bei dem an der Grenze der beiden Halbleiter zwei verschiedene Kristallgitter aufeinander treffen, so werden in einer Grenzschicht, für deren Dicke man etwa einige $10^{-7} \mathrm{~cm}$ ansetzen wird, außerordentlich starke Gitterstörungen herrschen. Diese Gitterstörungen wirken als Rekombinations-Generationszentren (Traps) und setzen die Lebensdauer der Stromträger stark herab. Ist die Lebensdauer hinreichend klein, so wird die Übernahme des Elektronenstromes durch den Defektelektronenstrom nur in dieser Grenzschicht stattfinden, und der durch die Anordnung fließende Strom wird praktisch im gesamten n-Leiter als reiner Elektronenstrom und im gesamten p-Leiter als reiner Defektelektronenstrom geführt (Abb. lc). Der innerhalb der Grenzschicht auftretende Rekombina-

21 W. Shockley, Bell System Tech. J. 28, 435 [1949]. tionsstrom $i_{\text {Rek }}$ muß gleich dem durch die Anordnung fließenden Gesamtstrom $i$ sein :

$$
i_{\mathrm{Rek}}=i \text {. }
$$

Betrachtet man das Energieschema des pn-Kontaktes (Abb. 2) in der nächsten Umgebung der Grenze zwischen den beiden Halbleitern, so wird man die Grenzschicht hoher Gitterstörungen einmal als das stark

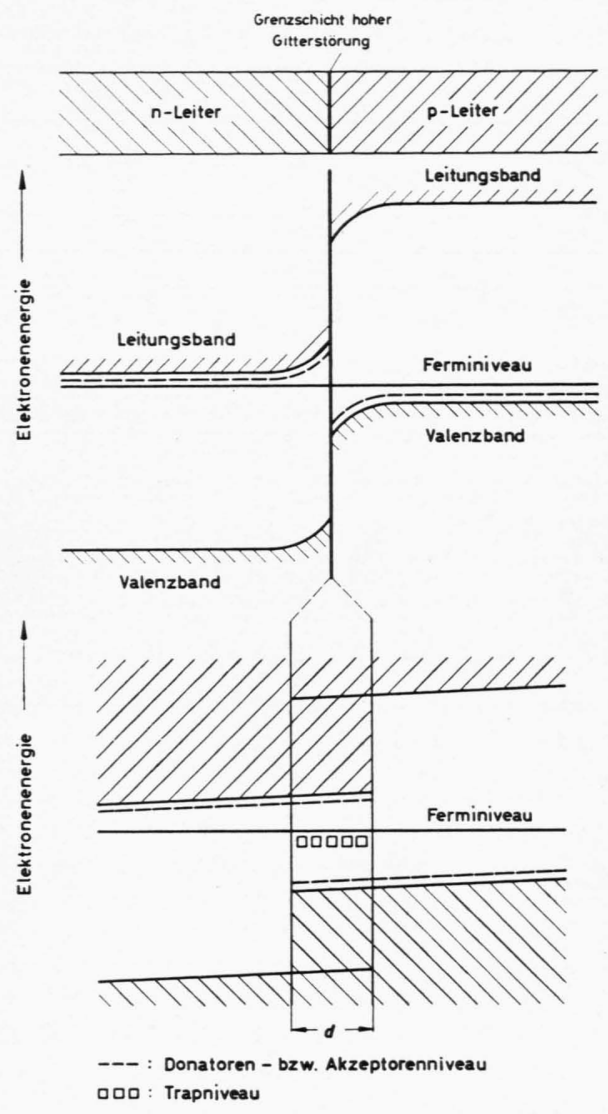

Abb. 2. Energieschema des pn-Kontaktes (thermisches Gleichgewicht).

gestörte Gitter des n-Leiters und zum anderen als das stark gestörte Gitter des p-Leiters ansehen können. Das bedeutet, daß sich innerhalb der Grenzschicht die Valenz- und Leitungsbänder der beiden Halbleiter überlappen und zahlreiche Trapniveaus existieren (von denen in Abb. 2 nur eines gezeichnet ist). Wegen der geringen Ausdehnung dieser Schicht kann man die Neigung der Bandkanten vernachlässigen und die Konzentrationen $n$ und $p$ der Stromträger als konstant ansehen. Bezeichnen wir diese „Randkonzentrationen" mit $n_{\mathrm{R}}$ und $p_{\mathrm{R}}$ und die Dicke der 
Schicht mit $d$, so ergibt sich für den Rekombinationsstrom $i_{\text {Rek }}$ nach Shockley und Read ${ }^{22}$ bei Berücksichtigung eines Trap-Niveaus:

$$
i_{\mathrm{Rek}}=e d \frac{\left(n_{\mathrm{R}} p_{\mathrm{R}}-n_{\mathrm{R}}^{0} p_{\mathrm{R}}^{0}\right)}{\left(n_{\mathrm{R}}+n_{1}\right) \tau_{\mathrm{po}}+\left(p_{\mathrm{R}}+p_{1}\right) \tau_{\mathrm{no}}}
$$

Dabei sind $e$ die elektrische Elementarladung, $n_{\mathrm{R}}{ }^{0}$ und $p_{\mathrm{R}}{ }^{0}$ die Randkonzentrationen im thermischen Gleichgewicht, $n_{\mathrm{R}}$ und $p_{\mathrm{R}}$ die zum Strome $i_{\mathrm{Rek}}$ gehörigen Werte der Randkonzentrationen, $n_{1}$ und $p_{1}$ die Randkonzentrationen für den Fall, daß das Fermi-Niveau mit dem Trap-Niveau zusammenfallen würde, und $\tau_{p_{0}}$ und $\tau_{\text {no }}$ die Lebensdauern von Defektelektronen im n-Leiter bzw. Elektronen im p-Leiter.

Die Forderung $i_{\mathrm{Rek}}=i$ stellt somit eine Bedingung für die Randkonzentrationen $n_{\mathrm{R}}$ und $p_{\mathrm{R}}$ in Abhängigkeit von dem durch die Anordnung fließenden Strom dar. Diese Bedingung wird besonders einfach, wenn wir annehmen, daß ein pn-Kontakt durch den Grenzfall verschwindend kleiner Lebensdauern $\tau_{\mathrm{po}}$ und $\tau_{\text {no }}$ innerhalb der Grenzschicht charakterisiert wird. Dann folgt nämlich aus (2.1) und (2.2), daß für jeden Wert des Stromes $i$ durch den pn-Kontakt die Abweichung des Produktes $n_{\mathrm{R}} p_{\mathrm{R}}$ von dem Wert $n_{\mathrm{R}}{ }^{0} p_{\mathrm{R}}{ }^{0}$ im thermischen Gleichgewicht verschwindend klein ist. Die Bedingung (2.1) wird somit zu

$$
n_{\mathrm{R}} p_{\mathrm{R}}=n_{\mathrm{R}}{ }^{0} p_{\mathrm{R}}{ }^{0},
$$

das Produkt $n_{\mathrm{R}} p_{\mathrm{R}}$ der Randkonzentrationen der Majoritätsträger der beiden Halbleiter ist unabhängig vom Strom.

Führt man noch die im n- bzw. p-Leiter auftretenden Diffusionsspannungen $V_{\mathrm{Dn}}$ und $V_{\mathrm{Dp}}$ ein, gemäß

$$
V_{\mathrm{Dn}}=\mathfrak{V} \ln \left(n_{\mathrm{H}} / n_{\mathrm{R}}\right), \quad V_{\mathrm{Dp}}=\mathfrak{V} \ln \left(p_{\mathrm{H}} / p_{\mathrm{R}}\right)
$$

$(\mathfrak{B}=k T / e ; k=$ Boltzmann-Konstante, $T=$ absolute Temperatur, $n_{\mathrm{H}}$ bzw. $p_{\mathrm{H}}=$,Neutraldichte“ der Elektronen im Inneren des n-Leiters bzw. der Defektelektronen im Inneren des p-Leiters), so kann man an Stelle von (2.3) auch schreiben

$$
V_{\mathrm{Dn}}+V_{\mathrm{Dp}}=V_{\mathrm{Dn}}^{0}+V_{\mathrm{Dp}}^{0}=V_{\mathrm{D}},
$$

wobei $V_{\mathrm{D}}$ die somit vom Strom unabhängige Diffusionsspannung des pn-Kontaktes ist.

Für die weiteren Betrachtungen wollen wir diese Bedingung (2.3) bzw. (2.3') als eine für einen pn-

22 W. Shockley u. W. T. Read, Phys. Rev. 87, 835 [1952].

23 Vgl. auch die Arbeit von O. Madelung, Zur Theorie des pnÜberganges bei verschwindender Lebensdauer der Ladungsträger, Z. Naturforschg. 12 a, 1020 [1957]. Hier wird die Gleichstromkennlinie unter Beschränkung auf den völlig
Kontakt charakteristische Randbedingung ansehen. Wenn die ihr zugrunde liegende Annahme verschwindend kleiner Lebensdauer der Stromträger in der Grenzschicht eines pn-Kontaktes in der Praxis auch nur näherungsweise erfüllt sein wird, so werden wir dennoch erwarten können, das charakteristische Verhalten eines pn-Kontaktes damit richtig zu beschreiben. Denn in jedem Falle wird die Lebensdauer der Stromträger in der Grenzschicht infolge der hohen Gitterstörungen wesentlich kleiner sein als im Inneren der beiden Halbleiter ${ }^{23}$.

\section{Die Grundgleichungen der Theorie}

Wir betrachten eine ebene Anordnung

Metall | Überschuß-Halbleiter | Defekt-Halbleiter Metall,

wobei die Halbleiter verschiedene Kristallgitter haben, und machen zunächst folgende allgemeine Annahmen:

A 1) Die beiden Metall-Halbleiter-Kontakte seien ohmisch.

A 2) Beide Halbleiter seien ausgesprochene Störstellenleiter; die Dichten der Minoritätsträger seien vernachlässigbar klein gegenüber den Dichten der Majoritätsträger.

A 3) Ionenleitung trete nicht auf; Ionenbewegungen spielen nur bei Formier- und Alterungserscheinungen eine Rolle.

A 4) An der Grenze der beiden Halbleiter sollen Verarmungsrandschichten auftreten.

A 5) Eine sich an der Grenze zwischen den beiden Halbleitern ausbildende atomare Doppelschicht sei unabhängig vom Strom.

Darüber hinaus seien noch die speziellen Annahmen erfüllt:

A 6) In beiden Halbleitern liege Nichtentartung vor.

A 7) Die Ausdehnungen der Halbleiter seien groß gegen die Dicken der Raumladungsschichten.

A 8) Die Störstellen seien in beiden Halbleitern vollständig dissoziiert.

A 9) Die Störstellendichte sei in beiden Halbleitern räumlich konstant.

symmetrischen pn-Übergang diskutiert, wobei die Annahme verschwindender Lebensdauer der Stromträger jedoch nicht nur für eine Grenzschicht, sondern für das gesamte Gebiet beider Halbleiter gemacht wird. 
Abb. 3 zeigt schematisch die Potentialverhältnisse und die Konzentrationsverteilung der Stromträger für eine derartige Anordnung. Dabei ist $x$ die Ortskoordinate in einer Richtung senkrecht zu der bei $x=0$ liegenden Grenze der beiden Halbleiter und

$L_{\mathrm{n}}, L_{\mathrm{p}} \quad=$ Dicke des n- bzw. p-Leiters,

$l_{\mathrm{n}}, l_{\mathrm{p}} \quad=$ Dicke der Raumladungsschicht im n- bzw. p-Leiter,

$V(x) \quad=$ Potential; $V=0$ für $x=L_{\mathrm{p}}$,

$U \quad=$ von außen angelegte Spannung,

$V_{\mathrm{D}} \quad=$ Diffusionsspannung des pn-Kontaktes,

$V_{\mathrm{DS}}=$ durch atomare Doppelschicht verursachter Potentialsprung,

$V_{\mathrm{p}}(x) \quad=V(x>0)=$ Potential im p-Leiter,

$V_{\mathrm{n}}(x)=V_{\mathrm{D}}+V_{\mathrm{DS}}+U-V(x<0)$,

$V_{\mathrm{D}_{\mathrm{n}}}+U_{\mathrm{n}}, V_{\mathrm{D}_{\mathrm{p}}}+U_{\mathrm{p}}=$ Potentialdifferenz über dem nbzw. p-Leiter,

$n(x), p(x)=$ Konzentration der Stromträger im n- bzw. p-Leiter,

$n_{\mathrm{R}}, p_{\mathrm{R}} \quad=$ Randkonzentrationen bei $x=0$,

$n_{\mathrm{D}}, n_{\mathrm{A}}=$ ortsunabhängige Störstellenkonzentration im n- bzw. p-Leiter.

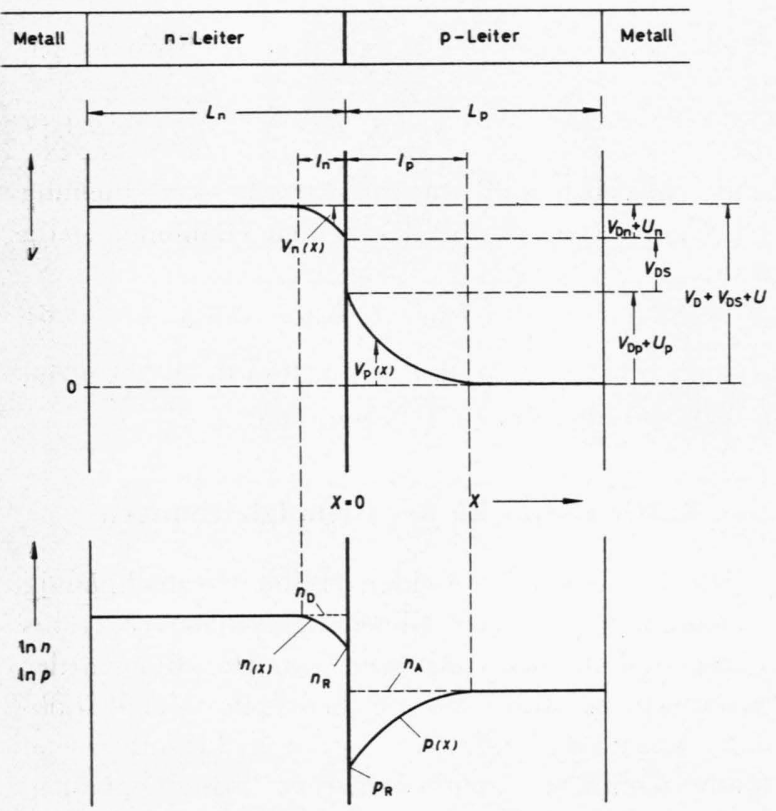

Abb. 3. Potentialverhältnisse und Konzentrationsverteilung der Stromträger.

Die Bahnfeldstärken innerhalb der raumladungsfreien Bereiche der Halbleiter und die über diesen Bereichen abfallenden Bahnspannungen wollen wir vernachlässigen. Eine spätere Berücksichtigung der Bahnspannungen ist - wie die genaue Rechnung ohne ihre Vernachlässigung zeigt - möglich, indem man in den erhaltenen Beziehungen die Spannung $U$ ersetzt durch

$U-i\left[\frac{\left(L_{\mathrm{n}}-l_{\mathrm{n}}\right)}{\sigma_{\mathrm{n}}}+\frac{\left(L_{\mathrm{p}}-l_{\mathrm{p}}\right)}{\sigma_{\mathrm{p}}}\right]$ oder wegen der Voraussetzung A 7 durch

$U-i\left[\frac{L_{\mathrm{n}}}{\sigma_{\mathrm{n}}}+\frac{L_{\mathrm{p}}}{\sigma_{\mathrm{p}}}\right]$, wobei $\sigma_{\mathrm{n}}$ und $\sigma_{\mathrm{p}}$ die Leitfähigkeiten der raumladungsfreien Halbleiter sind.

Im Inneren der Halbleiter sind die Konzentrationen der Stromträger gleich den Neutraldichten $n_{\mathrm{H}}$ bzw. $p_{\mathrm{H}}$, die wegen der vorausgesetzten vollständigen Dissoziation der Störstellen gleich den Störstellendichten $n_{\mathrm{D}}$ bzw. $n_{\mathrm{A}}$ sind. An der Grenze der Halbleiter bilden sich Verarmungsrandschichten, und die Dichten der Stromträger nehmen ab bis auf gewisse "Randkonzentrationen " $n_{\mathrm{R}}$ bzw $p_{\mathrm{R}}$, die zunächst unbekannt sind und von der angelegten Spannung $U$ abhängen können. Von der über der gesamten Anordnung auftretenden Potentialdifferenz $V_{\mathrm{D}}+V_{\mathrm{DS}}+U$ entfällt ein gewisser Teil $V_{\mathrm{D}_{\mathrm{n}}}+U_{\mathrm{n}}$ auf den n-Leiter, ein anderer Teil $V_{\mathrm{D}_{\mathrm{p}}}+U_{\mathrm{p}}$ auf den p-Leiter. Der Potentialsprung $V_{\text {DS }}$ ist der atomaren Doppelschicht an der Grenze der beiden Halbleiter zugeordnet und von $U$ unabhängig. Die Anteile $V_{\mathrm{D}_{\mathrm{n}}}, V_{\mathrm{D}_{\mathrm{p}}}, U_{\mathrm{n}}$ und $U_{\mathrm{p}}$ der Diffusionsspannung $V_{\mathrm{D}}$ und der von außen angelegten Spannung $U$ sind vorläufig ebenfalls unbekannt. Die Größen $V_{\mathrm{D}_{\mathrm{n}}}$ und $V_{\mathrm{D}_{\mathrm{p}}}$ sind jedoch durch die den Gln. (2.4) entsprechenden Beziehungen

$$
n_{\mathrm{R}}=n_{\mathrm{D}} \exp \left\{-V_{\mathrm{D}_{\mathrm{n}}} / \mathfrak{V}\right\} ; \quad p_{\mathrm{R}}=n_{\mathrm{A}} \exp \left\{-V_{\mathrm{D}_{\mathrm{p}}} / \mathfrak{V}\right\}
$$

mit den Randkonzentrationen $n_{\mathrm{R}}$ und $p_{\mathrm{R}}$ verknüpft.

Man erkennt, daß diese Anordnung zwei hintereinander geschalteten Metall-Halbleiter-Kontakten entspricht, wobei die "Randkonzentrationen " $n_{\mathrm{R}}$ und $p_{R}$ im allgemeinen aber von der angelegten Spannung abhängen werden. Gerade diese Spannungsabhängigkeit der Randkonzentrationen wird zu einer wesentlich anderen Gleichstromkennlinie führen, als sie sich für den Metall-Halbleiter-Kontakt nach Sсноттку ergibt. Für den völlig symmetrischen pnKontakt mit gleichen Parameterwerten der beiden homogenen Halbleitergebiete muß man jedoch erwarten, daß sich die Kennliniengleichung aus der von Sсноттку für den Metall-Halbleiter-Kontakt angegebenen ${ }^{24}$

$$
i=\frac{\mathfrak{V}}{R_{0}} \sqrt{1+\frac{U}{V_{\mathrm{D}}}}(1-\exp \{-U / \mathfrak{V}\})
$$

dadurch, daß man $U$ durch die an jedem der beiden Halbleiter liegenden Spannung $\frac{1}{2} U$, ebenso $V_{\mathrm{D}}$ durch

24 W. Sсноттку ${ }^{4}$, S. 547 , Gl. (13'). 
$\frac{1}{2} V_{\mathrm{D}}$ und den Nullwiderstand $R_{0}$ durch $\frac{1}{2} R_{0}$ ersetzt, $\mathrm{zu}$

$$
i=\frac{2 \mathfrak{B}}{R_{0}} \sqrt{1+\frac{U}{V_{\mathrm{D}}}}(1-\exp \{-U / 2 \mathfrak{V}\})
$$

ergibt.

Die Verhältnisse sind offenbar eindeutig bestimmt, wenn man die vier von der Spannung $U$ abhängigen Ortsfunktionen $n(x, U), p(x, U), V_{\mathrm{n}}(x, U)$ und $V_{\mathrm{p}}(x, U)$ angeben kann. Zur Ermittlung dieser Funktionen stehen für jeden der beiden Halbleiter die Porsson-Gleichung und die Kontinuitätsgleichung des Stromes zur Verfügung, ingesamt also die folgenden vier simultanen Differentialgleichungen ${ }^{25}$, welche die Grundgleichungen des Problems darstellen:

$$
\begin{gathered}
\frac{\mathrm{d}^{2} V_{\mathrm{n}}}{\mathrm{d} x^{2}}=\frac{4 \pi e}{\varepsilon_{\mathrm{n}} \varepsilon_{\mathrm{v}}}\left(n_{\mathrm{D}}-n\right) ; \frac{\mathrm{d}^{2} V_{\mathrm{p}}}{\mathrm{d} x^{2}}=\frac{4 \pi e}{\varepsilon_{\mathrm{p}} \varepsilon_{\mathrm{V}}}\left(n_{\mathrm{A}}-p\right) ; \\
i_{\mathrm{n}}=e b_{\mathrm{n}} n E_{\mathrm{n}}+e b_{\mathrm{n}} \mathfrak{V} \frac{\mathrm{d} n}{\mathrm{~d} x} ; \\
i_{\mathrm{p}}=e b_{\mathrm{p}} p E_{\mathrm{p}}-e b_{\mathrm{p}} \mathfrak{V} \frac{\mathrm{d} p}{\mathrm{~d} x} .
\end{gathered}
$$

Dabei gilt:

$\varepsilon_{\mathrm{n}}, \varepsilon_{\mathrm{p}}=$ relative Dielektrizitätskonstante des n- bzw. p-Leiters,

$\varepsilon_{\mathrm{v}}=$ Dielektrizitätskonstante des Vakuums ${ }^{25}$,

$i_{\mathrm{n}}, i_{\mathrm{p}}=$ Stromdichte im n- bzw. p-Leiter, positiv in $x$-Richtung,

$b_{\mathrm{n}}, b_{\mathrm{p}}=$ Beweglichkeit der Stromträger im n- bzw. pLeiter,

$E_{\mathrm{n}}=-\left(\frac{\mathrm{d} V}{\mathrm{~d} x}\right)_{x \leqq 0}=+\frac{\mathrm{d} V_{\mathrm{n}}}{\mathrm{d} x}=$ Feldstärke im n-Leiter,

$E_{\mathrm{p}}=-\left(\frac{\mathrm{d} V}{\mathrm{~d} x}\right)_{x \geqq 0}=-\frac{\mathrm{d} V_{\mathrm{p}}}{\mathrm{d} x}=$ Feldstärke im p-Leiter.

Es ist nun zweckmäßig, dieses System von 4 simultanen Differentialgleichungen aufzuspalten in zwei voneinander unabhängige Paare von simultanen Differentialgleichungen, indem man die unbekannten Teilspannungen $V_{\mathrm{D}_{\mathrm{n}}}, V_{\mathrm{D}_{\mathrm{p}}}, U_{\mathrm{n}}$ und $U_{\mathrm{p}}$ als gegeben ansieht und die sechs zu dem obigen Gleichungssystem gehörigen Randbedingungen folgendermaßen formuliert (s. Abb. 3) :

$$
\begin{aligned}
& V_{\mathrm{n}}\left(x \leqq-l_{\mathrm{n}}\right)=0 ; \quad V_{\mathrm{p}}\left(x \geqq+l_{\mathrm{p}}\right)=0 ; \\
& V_{\mathrm{n}}(x=0) \quad=V_{\mathrm{D}_{\mathrm{n}}}+U_{\mathrm{n}} ; V_{\mathrm{p}}(x=0) \quad=V_{\mathrm{D}_{\mathrm{p}}}+U_{\mathrm{p}} ; \\
& n\left(x \leqq-l_{\mathrm{n}}\right)=n_{\mathrm{D}} ; \quad p\left(x \geqq l_{\mathrm{p}}\right) \quad=n_{\mathrm{A}} \text {. }
\end{aligned}
$$

Damit sind die für den n-Leiter geltenden Gleichungen unabhängig von den für den p-Leiter gel-

25 Sämtliche Gleichungen sind durch die Einführung der Größe $\varepsilon_{\mathrm{V}}$ für die Dielektrizitätskonstante des Vakuums in einer vom verwendeten Maßsystem unabhängigen Form tenden. Aus den Gln. (3.2) bis (3.4) sind einerseits die Funktionen $n(x)$ und $V_{\mathrm{n}}(x)$ in Abhängigkeit von den Parametern $V_{\mathrm{D}_{\mathrm{n}}}$ und $U_{\mathrm{n}}$, andererseits die Funktionen $p(x)$ und $V_{\mathrm{p}}(x)$ in Abhängigkeit von $V_{\mathrm{Dp}_{\mathrm{p}}}$ und $U_{\mathrm{p}}$ zu bestimmen (Abschn. 4).

Zur endgültigen Lösung des Problems hat man dann die Größen $V_{\mathrm{D}_{\mathrm{n}}}, V_{\mathrm{D}_{\mathrm{p}}}, U_{\mathrm{n}}$ und $U_{\mathrm{p}}$ als Funktionen der angelegten Spannung $U$ zu ermitteln. Hierzu stehen die folgenden vier Randbedingungen des Systems zur Verfügung:

Erstens muß die in Abschn. 2 ermittelte charakteristische Randbedingung (2.3') des pn-Kontaktes erfüllt werden,

$$
V_{\mathrm{D}_{\mathrm{n}}}+V_{\mathrm{Dp}_{\mathrm{p}}}=V_{\mathrm{D}} \cdot
$$

Zweitens muß die Summe aller Teilspannungen gleich der über der gesamten Anordnung auftretenden Potentialdifferenz sein, d. h. (s. Abb. 3)

$$
\begin{aligned}
V_{\mathrm{D}_{\mathrm{n}}}+U_{\mathrm{n}}+V_{\mathrm{DS}}+V_{\mathrm{D}_{\mathrm{p}}}+U_{\mathrm{p}} & =V_{\mathrm{D}}+V_{\mathrm{DS}}+U, \\
\text { oder wegen }(3.5) \quad U_{\mathrm{n}}+U_{\mathrm{p}} & =U .
\end{aligned}
$$

Weiter muß wegen der Kontinuität des Stromes gelten

$$
i_{\mathrm{n}}=i_{\mathrm{p}},
$$

und schließlich muß die dielektrische Verschiebung $\varepsilon E$ an der Grenzfläche der beiden Halbleiter stetig sein:

$$
\varepsilon_{\mathrm{n}} E_{\mathrm{nR}}=\varepsilon_{\mathrm{p}} E_{\mathrm{pR}},
$$

wenn wir mit $E_{\mathrm{nR}}$ und $E_{\mathrm{pR}}$ die Randfeldstärken im n- bzw. p-Leiter bei $x=0$ bezeichnen.

\section{Die Lösungen der Grundgleichungen}

Die für jeden der beiden Halbleiter unabhängig voneinander geltenden Gleichungssysteme (3.2) bis (3.4) sind identisch mit den Grundgleichungen der Sснотткуschen Raumladungstheorie des Metall-Halbleiter-Kontaktes ${ }^{1,2,4}$. Ihre strenge Lösung bietet unüberwindliche mathematische Schwierigkeiten. Sсноттку $^{4}$ und Spenke ${ }^{5}$ trennen den Zusammenhang zwischen den Gln. (3.2) und (3.3) deshalb dadurch auf, daß sie den Einfluß der Konzentration $n$ bzw. $p$ der Stromträger auf die Raumladungsdichte innerhalb der Raumladungsschicht als sehr gering ansehen. Sie vernachlässigen daher in der Gl. (3.2) $n$ gegenüber $n_{\mathrm{D}}$ bzw. $p$ gegenüber $n_{\mathrm{A}}$.

geschrieben. Im praktischen Maßsystem ist $\varepsilon_{\mathrm{V}}=4 \pi \varepsilon_{0}$ mit $\varepsilon_{0}=8,859 \cdot 10^{-14} \mathrm{Asec} / \mathrm{Vcm}$, im elektrostatischen Maßsystem gilt $\varepsilon_{\mathrm{V}}=1$ (elektrostat. Ladungseinheit) ${ }^{2} / \mathrm{erg} / \mathrm{cm}$. 
Hierdurch läßt sich aus (3.2) der Potentialverlauf und danach aus (3.3) die Konzentration $n$ bzw. $p$ der Stromträger als Funktion des Ortes $x$ ermitteln.

Es wird sich aber zeigen, daß zur Bestimmung der wesentlichen Eigenschaften des pn-Kontaktes die Kenntnis der Ortsfunktionen $n(x), p(x), V_{\mathrm{n}}(x)$ und $V_{\mathrm{p}}(x)$ nicht notwendig ist. Vielmehr genügt es, die Abhängigkeit der Stromträgerkonzentrationen und der Feldstärke vom Potential $V$ zu kennen. Wir gehen daher von der Variablen $x$ über zur Variablen $V$ bzw. $V_{\mathrm{n}}$ oder $V_{\mathrm{p}}$. Dann lauten die Gln. (3.2) und (3.3) :

$$
\begin{aligned}
& \frac{\mathrm{d} E_{\mathrm{n}}^{2}}{\mathrm{~d} V_{\mathrm{n}}}=\frac{8 \pi e}{\varepsilon_{\mathrm{n}} \varepsilon_{\mathrm{V}}}\left(n_{\mathrm{D}}-n\right) ; \\
& \frac{\mathrm{d} E_{\mathrm{p}}{ }^{2}}{\mathrm{~d} V_{\mathrm{p}}}=\frac{8 \pi e}{\varepsilon_{\mathrm{p}} \varepsilon_{\mathrm{V}}}\left(n_{\mathrm{A}}-p\right) ; \\
& i_{\mathrm{n}}=e b_{\mathrm{n}} E_{\mathrm{n}}\left(n+\mathfrak{V} \frac{\mathrm{d} n}{\mathrm{~d} V_{\mathrm{n}}}\right) ; \\
& i_{\mathrm{p}}=e b_{\mathrm{p}} E_{\mathrm{p}}\left(p+\mathfrak{V} \frac{\mathrm{d} p}{\mathrm{~d} V_{\mathrm{p}}}\right) .
\end{aligned}
$$

Die zugehörigen Nebenbedingungen lauten jetzt:

$$
\begin{aligned}
E_{\mathrm{n}}\left(V_{\mathrm{n}}=0\right) & =0 ; \quad E_{\mathrm{p}}\left(V_{\mathrm{p}}=0\right)=0 ; \\
n\left(V_{\mathrm{n}}=0\right) & =n_{\mathrm{D}} ; \quad p\left(V_{\mathrm{p}}=0\right)=n_{\mathrm{A}} .
\end{aligned}
$$

Die Grundgleichungen (4.1) und (4.2) stellen zwei sich völlig entsprechende Paare von simultanen Differentialgleichungen dar. Es genügt daher, im folgenden ihre Lösung z. B. nur für den p-Leiter zu verfolgen. Für den n-Leiter gelten jeweils entsprechende Beziehungen.

Die Lösung von Gl. (4.2) lautet

$$
\begin{aligned}
& p\left(V_{\mathrm{p}}\right)=n_{\mathrm{A}} \exp \left\{-V_{\mathrm{p}} / \mathfrak{B}\right\} \\
& +\frac{i_{\mathrm{p}} \exp \left\{-V_{\mathrm{p}} / \mathfrak{B}\right\}}{e b_{\mathrm{p}} \mathfrak{O}} \int_{0}^{V_{\mathrm{p}}} E_{\mathrm{p}}{ }^{-1} \cdot \exp \left\{+V_{\mathrm{p}} / \mathfrak{B}\right\} \mathrm{d} V_{\mathrm{p}},
\end{aligned}
$$

woraus sich für $V_{\mathrm{p}}=V_{\mathrm{Dp}}+U_{\mathrm{p}}$ unter Beachtung von (3.1) ergibt

$$
i_{\mathrm{p}}=e b_{\mathrm{p}} n_{\mathrm{A}} \mathfrak{\mathfrak { B }} \frac{\left(\exp \left\{U_{\mathrm{p}} / \mathfrak{B}\right\}-1\right)}{\int_{0}^{V_{\mathrm{Dp}}+U_{\mathrm{p}}} E_{\mathrm{p}}^{-1} \cdot \exp \left\{V_{\mathrm{p}} / \mathfrak{B}\right\} \mathrm{d} V_{\mathrm{p}}} .
$$

Bei Verwendung der durch (4.5) gegebenen Abhängigkeit der Defektelektronenkonzentration vom Potential erhält man nunmehr eine Lösung der PoissonGleichung (4.1), aus der sich (bei Vernachlässigung der Bahnfeldstärke und der Bahnspannung) folgender Ausdruck für die Randfeldstärke $E_{\mathrm{pR}}$ an der Stelle $x=0$ ergibt:

$$
\begin{aligned}
E_{\mathrm{pR}}^{2}= & \frac{8 \pi e n_{\mathrm{A}}}{\varepsilon_{\mathrm{p}} \varepsilon_{\mathrm{v}}} \\
& \cdot\left[V_{\mathrm{D}_{\mathrm{p}}}+U_{\mathrm{p}}-\mathfrak{B}\left(1-\exp \left\{-V_{\mathrm{D}_{\mathrm{p}}} / \mathfrak{B}\right\}\right)\right] .
\end{aligned}
$$

Damit sind sämtliche in den Randbedingungen (3.5) bis (3.8) auftretenden Größen als Funktionen der Parameter $V_{\mathrm{D}_{\mathrm{n}}}, V_{\mathrm{D}_{\mathrm{p}}}, U_{\mathrm{n}}$ und $U_{\mathrm{p}}$ bestimmt, und die Bestimmungsgleichungen für diese Parameter lauten somit:

$$
\begin{gathered}
V_{\mathrm{D}_{\mathrm{n}}}+V_{\mathrm{D}_{\mathrm{p}}}=V_{\mathrm{D}}, \quad U_{\mathrm{n}}+U_{\mathrm{p}}=U, \quad(4.8,9) \\
b_{\mathrm{n}} n_{\mathrm{D}} \frac{\left(\exp \left\{U_{\mathrm{n}} / \mathfrak{B}\right\}-1\right)}{\int_{0}^{V_{\mathrm{Dn}}+U_{\mathrm{n}}} E_{\mathrm{n}}-1 \cdot \exp \left\{V_{\mathrm{n}} / \mathfrak{B}\right\} \mathrm{d} V_{\mathrm{n}}} \\
=b_{\mathrm{p}} n_{\mathrm{A}} \frac{\left(\exp \left\{U_{\mathrm{p}} / \mathfrak{B}\right\}-1\right)}{\int_{0}^{V_{\mathrm{p} p}+U_{\mathrm{p}}} E_{\mathrm{n}}^{-1} \cdot \exp \left\{V_{\mathrm{p}} / \mathfrak{B}\right\} \mathrm{d} V_{\mathrm{p}}} ; \\
\varepsilon_{\mathrm{n}} n_{\mathrm{D}}\left(\frac{V_{\mathrm{D}_{\mathrm{n}}}+U_{\mathrm{n}}}{\mathfrak{B}}-1+\exp \left\{-V_{\mathrm{D}_{\mathrm{n}}} / \mathfrak{B}\right\}\right) \\
=\varepsilon_{\mathrm{p}} n_{\mathrm{A}}\left(\frac{V_{\mathrm{D}_{\mathrm{p}}}+U_{\mathrm{p}}}{\mathfrak{V}}-1+\exp \left\{-V_{\mathrm{D}_{\mathrm{p}}} / \mathfrak{B}\right\}\right) .
\end{gathered}
$$

Dieses Gleichungssystem ist nicht mehr exakt lösbar, insbesondere weil man zur Auswertung der in (4.10) auftretenden Integrale auf Näherungen angewiesen ist. Wir werden seine Lösung daher für verschiedene Fälle diskutieren. Zuvor wollen wir jedoch die als Konstante des Gleichungssystems auftretende Diffusionsspannung $V_{\mathrm{D}}$ betrachten.

\section{Die Diffusionsspannung $V_{\mathrm{D}}$}

Abb. 4 zeigt das Energieschema des stromlosen pn-Kontaktes auf Grund des Bändermodells. Man liest daraus unschwer die Beziehung ab

$$
\begin{aligned}
-e\left(V_{\mathrm{D}_{\mathrm{n}}}+V_{\mathrm{D}_{\mathrm{p}}}+V_{\mathrm{DS}}\right) & =-e\left(V_{\mathrm{D}}+V_{\mathrm{DS}}\right) \\
& =E_{\mathrm{V}_{\mathrm{p}}}-\zeta_{\mathrm{p}}-\zeta_{\mathrm{n}}-E_{\mathrm{C}_{\mathrm{n}}},
\end{aligned}
$$

woraus sich für die Diffusionsspannung ergibt:

$$
V_{\mathrm{D}}=\frac{E_{\mathrm{C}_{\mathrm{n}}}-E_{\mathrm{V}_{\mathrm{p}}}-e V_{\mathrm{DS}}}{e}-\frac{k T}{e} \ln \frac{N_{\mathrm{C}_{\mathrm{n}}} N_{\mathrm{V}_{\mathrm{p}}}}{n_{\mathrm{D}}} \cdot
$$

da weitab von der Grenze der beiden Halbleiter $n=n_{\mathrm{D}}$ bzw. $p=n_{\mathrm{A}}$ und somit $\zeta_{\mathrm{n}}=-k T \ln \left(N_{\mathrm{C}_{\mathrm{n}}} / n_{\mathrm{D}}\right)$ und $\zeta_{\mathrm{p}}=-k T \ln \left(N_{\mathrm{V}_{\mathrm{p}}} / n_{\mathrm{A}}\right)$ gilt.

Damit ist gezeigt, wie die Diffusionsspannung von den Kristallenergien, den effektiven Zustandsdichten und den Störstellendichten der Halbleiter abhängt. Eine Vorausberechnung für ein bestimmtes Halbleiterpaar ist jedoch nicht möglich, da man die Kristallenergien im Valenz- und Leitungsband einzeln nicht kennt und die Größe des durch eine atomare Doppelschicht verursachten Potentialsprunges

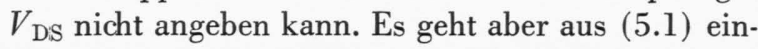




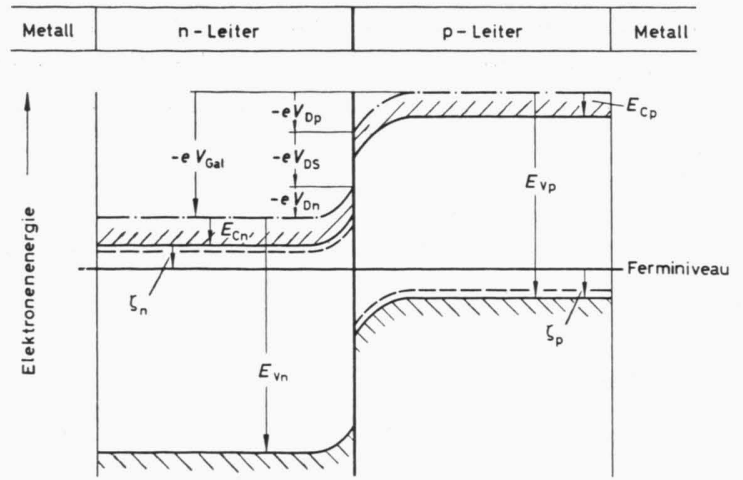

Abb. 4. Energieschema des stromlosen pn-Kontaktes.

$-\cdot-\cdot-$ : $-e \times$ Makropotential,

/////: Unterkante des Leitungsbandes,

$\backslash \backslash \backslash \backslash \backslash \backslash:$ Oberkante des Valenzbandes,

_____ : Störstellenniveau,

$V_{\mathrm{Gal}}=\mathrm{G}_{\mathrm{ALVANI}}$-Spannung,

$V_{\mathrm{DS}}=$ Potentialsprung durch atomare Doppelschicht,

$V_{\mathrm{D}}=V_{\mathrm{D}_{\mathrm{n}}}+V_{\mathrm{D}_{\mathrm{p}}}=$ Diffusionsspannung,

$E_{\mathrm{c}_{\mathrm{n}}}, E_{\mathrm{c}_{\mathrm{p}}}=$ Kristallenergie eines Elektrons am unteren Rande des Leitungsbandes,

$E_{\mathrm{v}_{\mathrm{n}}}, E_{\mathrm{v}_{\mathrm{p}}}=$ Kristallenergie eines Elektrons am oberen Rande des Valenzbandes,

$\zeta_{\mathrm{n}}=-k T \ln \left(N_{\mathrm{c}_{\mathrm{n}}} / n\right), \quad \zeta_{\mathrm{p}}=-k T \ln \left(N_{\mathrm{v}_{\mathrm{p}}} / p\right)$

(für Nicht-

$N_{\mathrm{c}_{\mathrm{n}}}, N_{\mathrm{v}_{\mathrm{p}}}=$ effektive Zustandsschichten,

$n, p=$ Dichten der Majoritätsträger.

deutig hervor, daß die Diffusionsspannung mit steigender Temperatur abnimmt, und daß sie mit steigender Störstellendichte zunimmt, und zwar bei einer Zunahme des Produktes $n_{\mathrm{D}} \cdot n_{\mathrm{A}}$ der Störstellendichten im n- und p-Leiter um den Faktor 10 um $\Delta V_{\mathrm{D}}=(k T / e) \ln 10$ (das sind bei Zimmertemperatur etwa $60 \mathrm{mV}$ ).

\section{Der stromlose pn-Kontakt}

Für den Fall des thermischen Gleichgewichtes reduziert sich das Gleichungssystem (4.8) bis (4.11) wegen $i=0, U=0$ auf die beiden Beziehungen

$$
\begin{gathered}
V_{\mathrm{D}_{\mathrm{n}}^{0}}^{0}+V_{\mathrm{D}_{\mathrm{p}}^{0}}^{0}=V_{\mathrm{D}}, \\
\varepsilon_{\mathrm{n}} n_{\mathrm{D}}\left(V_{\mathrm{D}_{\mathrm{n}}^{0}}^{0} / \mathfrak{P}-1+\exp \left\{-V_{\mathrm{D}_{\mathrm{n}}^{0}}^{0} / \mathfrak{P}\right\}\right) \\
=\varepsilon_{\mathrm{p}} n_{\mathrm{A}}\left(V_{\mathrm{D}_{\mathrm{p}}^{0}}^{0} / \mathfrak{P}-1+\exp \left\{-V_{\mathrm{D}}^{0} / \mathfrak{B}\right\}\right),
\end{gathered}
$$

wobei wir die Werte von spannungsabhängigen Größen für $U=0$ durch einen hochgestellten Index ${ }^{\prime \prime}$ “ kennzeichnen. Hieraus sind die Teildiffusionsspannungen $V_{\mathrm{D}}^{0}$ und $V_{\mathrm{D}_{\mathrm{p}}}^{0}$ bzw. die Randkonzentrationen $n_{\mathrm{R}}{ }^{0}$ und $p_{\mathrm{R}}{ }^{0}$ [s. Gl. (3.1)] im thermischen Gleichgewicht zu bestimmen.
Für das Produkt der Randkonzentrationen, welches nach Abschn. 2 stromunabhängig ist, ergibt sich mit Gl. (5.1)

$$
\begin{aligned}
n_{\mathrm{R}} p_{\mathrm{R}} & =n_{\mathrm{R}}^{0} p_{\mathrm{R}}^{0}=n_{\mathrm{D}} n_{\mathrm{A}} \exp \left\{-V_{\mathrm{D}} / \mathfrak{B}\right\} \\
& =N_{\mathrm{C}_{\mathrm{n}}} N_{\mathrm{V}_{\mathrm{p}}} \exp \frac{-\left(E_{C_{\mathrm{n}}}-E_{\mathrm{V}_{\mathrm{p}}}-e V_{\mathrm{DS}}\right)}{k T} .
\end{aligned}
$$

Hieraus geht hervor, daß das Produkt der Randkonzentrationen eines pn-Kontaktes nicht nur stromunabhängig, sondern auch unabhängig von den Störstellendichten $n_{\mathrm{D}}$ und $n_{\mathrm{A}}$ der beiden Halbleiter ist.

Die Gln. (6.1) und (6.2) lassen sich nicht geschlossen, sondern nur numerisch lösen. Das Ergebnis der Rechnung zeigt das in Abb. 5 dargestellte Nomogramm, dem man die relativen Randkonzentrationen $n_{\mathrm{R}}{ }^{0} / n_{\mathrm{D}}$ und $p_{\mathrm{R}}{ }^{0} / n_{\mathrm{A}}$ für den stromlosen pn-

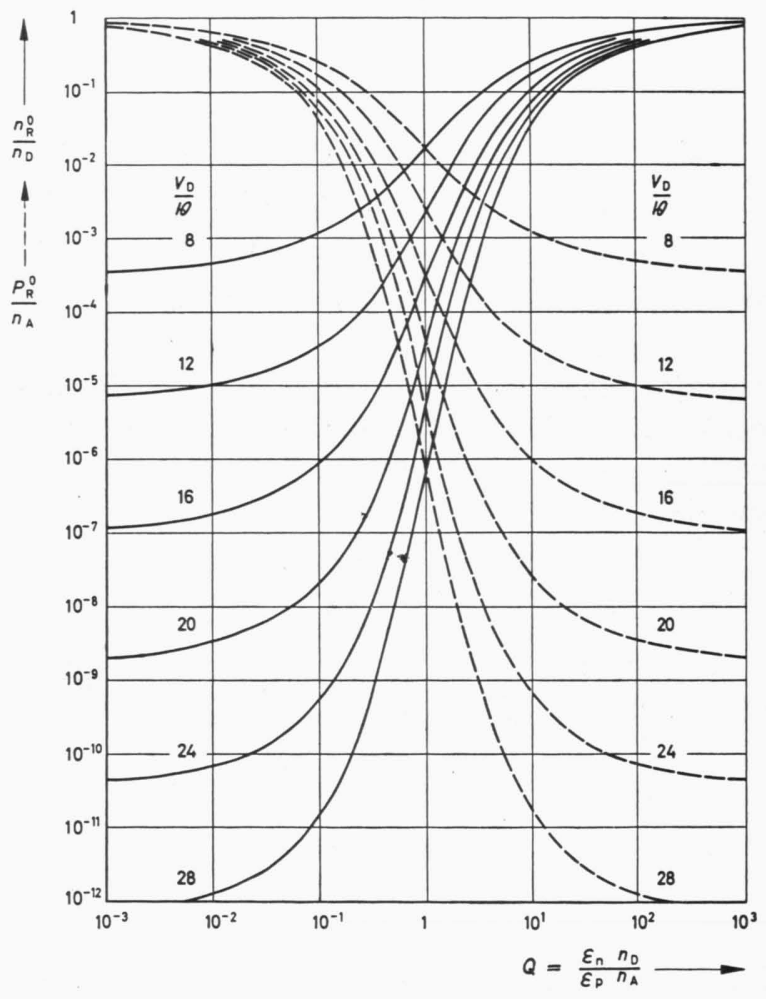

Abb. 5. Nomogramm zur Bestimmung der Randkonzentrationen $n_{\mathrm{R}}^{0}$ und $p_{\mathrm{R}}{ }^{0}$ des stromlosen pn-Kontaktes. $n_{\mathrm{D}}, n_{\mathrm{A}}=$ Störstellendichte im n- bzw. p-Leiter, $\varepsilon_{\mathrm{n}}, \varepsilon_{\mathrm{p}}=$ Dielektrizitätskonstante des n- bzw. p-Leiters, $V_{D}=$ Diffusionsspannung des pnKontaktes, $\mathfrak{B}=k T / e$. Die Bestimmung der Randkonzentrationen $\mathrm{nR}^{0}$ und $\mathrm{pR}^{0}$ aus dem Nomogramm setzt die Kenntnis der Diffusionsspannung und des Verhältnisses $\varepsilon_{\mathrm{n}} n_{\mathrm{D}} / \varepsilon_{\mathrm{p}} n_{\mathrm{A}}$ voraus. Will man die Änderung der Randkonzentrationen bei Variation einer oder beider Störstellendichten ermitteln, so hat man zu beachten, daß durch eine solche Variation auch die Diffusionsspannung $V_{\mathrm{D}}$ geändert wird! Diese Änderung läßt sich nach Abschn. 5 mit Gl. (5.1) berechnen. 
Kontakt in Abhängigkeit von der Diffusionsspannung $V_{\mathrm{D}}$ und dem Verhältnis der Störstellendichten entnehmen kann.

Die relativen Randkonzentrationen im thermischen Gleichgewicht hängen somit außer von der Diffusionsspannung $V_{\mathrm{D}}$ nur noch von dem Verhältnis

$$
Q=\varepsilon_{\mathrm{n}} n_{\mathrm{D}} / \varepsilon_{\mathrm{p}} n_{\mathrm{A}}
$$

$\mathrm{ab}$, aber nicht von den Beweglichkeiten der Stromträger. Für $Q=1$ sind auch die relativen Randkonzentrationen in beiden Halbleitern gleich. Ist jedoch $Q>1$, so ist $n_{\mathrm{R}}{ }^{0}$ um mehrere Größenordnungen größer als $p_{\mathrm{R}}{ }^{0}$, und umgekehrt.

\section{Der stromdurchflossene pn-Kontakt: Die Näherungen zur Lösung des Gleichungs- systems (4.8) bis (4.11)}

Um zu einer Lösung des Gleichungssystems (4.8) bis (4.11) zu kommen, machen wir die Voraussetzungen

$$
\begin{aligned}
V_{\mathrm{D}} / \mathfrak{B} & \gg 1 . \\
\left(V_{\mathrm{D}}+U\right) / \mathfrak{B} & \gg 1 .
\end{aligned}
$$

Die erste Voraussetzung (7.1) bedeutet, daß wir nur solche pn-Kontakte betrachten, die eine Diffusionsspannung von einigen Zehntel Volt und damit Randschichten mit einer nennenswerten Randverarmung an Stromträgern aufweisen. Für die Praxis ist dies kaum eine Einschränkung, da nur solche Fälle ausgeschlossen werden, in denen infolge geringer Randverarmung keine wesentlichen Gleichrichtereffekte auftreten.
Die Voraussetzung (7.2) neben (7.1) beschränkt unsere Betrachtungen auf nicht zu hohe Belastungen des pn-Kontaktes in Durchlaßrichtung $(U<0)$. Wir erhalten damit aus den Gln. (4.8), (4.9) und (4.11) für die Aufteilung der Potentialdifferenz $V_{\mathrm{D}}+U$ auf die beiden Halbleiter

$$
V_{\mathrm{D}_{\mathrm{p}}}+U_{\mathrm{p}}=\frac{V_{\mathrm{D}}+U}{1+Q^{-1}}, \quad V_{\mathrm{D}_{\mathrm{n}}}+U_{\mathrm{n}}=\frac{V_{\mathrm{D}}+U}{1+Q},
$$

und für die Randfeldstärken mit (3.8) und (4.7)

$\varepsilon_{\mathrm{p}} E_{\mathrm{pR}}=\varepsilon_{\mathrm{n}} E_{\mathrm{nR}}=\sqrt{\frac{8 \pi e}{\varepsilon_{\mathrm{V}}}} \sqrt{\frac{V_{\mathrm{D}}+U}{\mathrm{l} / \varepsilon_{\mathrm{n}} n_{\mathrm{D}}+1 / \varepsilon_{\mathrm{p}} n_{\mathrm{A}}}}$.

Für die in Gl. (4.10) auftretenden Integrale kann man schließlich folgende Näherungen ableiten:

$$
\begin{aligned}
& \int_{0}^{V_{\mathrm{p}}+U_{\mathrm{p}}} E_{\mathrm{p}}-1 \exp \left\{V_{\mathrm{p}} / \mathfrak{V}\right\} \mathrm{d} V_{\mathrm{p}} \\
& \left.=a_{\mathrm{p}}\left(\mathfrak{B} / E_{\mathrm{pR}}\right) \exp \left\{\left(V_{\mathrm{D}_{\mathrm{p}}}+U_{\mathrm{p}}\right) / \mathfrak{B}\right\}-1\right) \\
& \text { mit } \quad a_{\mathrm{p}}=1 \text { für }\left(V_{\mathrm{D}_{\mathrm{p}}}+U_{\mathrm{p}}\right) / \mathfrak{V} \gg 1, \\
& \text { und } \quad a_{\mathrm{p}}>2 \text { für }\left(V_{\mathrm{D}_{\mathrm{p}}}+U_{\mathrm{p}}\right) / \mathfrak{V} \ll 1 .
\end{aligned}
$$

Entsprechende Beziehungen gelten für das über das Potential $V_{\mathrm{n}}$ im n-Leiter erstreckte Integral.

\section{Die Spannungsabhängigkeit der Rand- konzentrationen}

Mit Hilfe der im vorigen Abschnitt angegebenen Näherungen (7.3) und (7.5) läßt sich nunmehr das Gleichungssystem (4.8) bis (4.11) für die Teilspannungen $V_{\mathrm{D}_{\mathrm{n}}}, V_{\mathrm{D}_{\mathrm{p}}}, U_{\mathrm{n}}$ und $U_{\mathrm{p}}$ lösen. Hier wollen wir zunächst nur die Teildiffusionsspannungen $V_{\mathrm{D}_{\mathrm{n}}}$ und $V_{\mathrm{D}_{\mathrm{p}}}$, d. h. die Randkonzentrationen $n_{\mathrm{R}}$ und $p_{\mathrm{R}}$ betrachten. Die Rechnung ergibt

$$
\begin{aligned}
\frac{p_{\mathrm{R}}}{n_{\mathrm{A}}}= & \exp \left\{-V_{\mathrm{D}} / \mathfrak{B}\right\} \\
= & \frac{1}{2}\left[\exp \left\{-\frac{V_{\mathrm{D}}+U}{\mathfrak{B}\left(1+Q^{-1}\right)}\right\}-\frac{b_{\mathrm{n}} n_{\mathrm{D}} \varepsilon_{\mathrm{p}} a_{\mathrm{p}}}{b_{\mathrm{p}} n_{\mathrm{A}} \varepsilon_{\mathrm{n}} a_{\mathrm{n}}} \exp \left\{-\frac{V_{\mathrm{D}}+U}{\mathfrak{B}(1+Q)}\right\} \cdot \frac{1-\exp \left\{-\left(V_{\mathrm{D}}+U\right) / \mathfrak{B}\left(1+Q^{-1}\right)\right\}}{1-\exp \left\{-\left(V_{\mathrm{D}}+U\right) / \mathfrak{B}(1+Q)\right\}}\right] \\
+\left\{\frac { 1 } { 4 } \left[\exp \left\{-\frac{V_{\mathrm{D}}+U}{\mathfrak{B}\left(1+Q^{-1}\right)}\right\}\right.\right. & \left.+\frac{b_{\mathrm{n}} n_{\mathrm{D}} \varepsilon_{\mathrm{p}} a_{\mathrm{p}}}{b_{\mathrm{p}} n_{\mathrm{A}} \varepsilon_{\mathrm{n}} a_{\mathrm{n}}} \exp \left\{-\frac{V_{\mathrm{D}}+U}{\mathfrak{B}(1+Q)}\right\} \cdot \frac{1-\exp \left\{-\left(V_{\mathrm{D}}+U\right) / \mathfrak{B}\left(1+Q^{-1}\right)\right\}}{1-\exp \left\{-\left(V_{\mathrm{D}}+U\right) / \mathfrak{B}(1+Q)\right\}}\right]^{2} \\
& \left.+\frac{b_{\mathrm{n}} n_{\mathrm{D}} \varepsilon_{\mathrm{p}} a_{\mathrm{p}}}{b_{\mathrm{p}} n_{\mathrm{A}} \varepsilon_{\mathrm{n}} a_{\mathrm{n}}} \cdot \frac{1-\exp \left\{-\left(V_{\mathrm{D}}+U\right) / \mathfrak{B}\left(1+Q^{-1}\right)\right\}}{1-\exp \left\{-\left(V_{\mathrm{D}}+U\right) / \mathfrak{B}(1+Q)\right\}} \cdot \frac{1-\exp \{-U / \mathfrak{B}\}}{\exp \left\{V_{\mathrm{D}} / \mathfrak{B}\right\}}\right\}^{1 / 2},
\end{aligned}
$$

$$
\begin{aligned}
n_{\mathrm{R}} / n_{\mathrm{D}} & =\exp \left\{-V_{\mathrm{D}_{\mathrm{n}}} / \mathfrak{P}\right\} \\
& =\exp \left\{-V_{\mathrm{D}} / \mathfrak{B}\right\} \cdot \exp \left\{+V_{\mathrm{D}_{\mathrm{p}}} / \mathfrak{V}\right\} .
\end{aligned}
$$

Man erkennt hieraus, daß die Randkonzentrationen im allgemeinen von der von außen angelegten Spannung $U$ abhängen, wobei außer den Störstellen- dichten auch die Beweglichkeiten der Stromträger eine Rolle spielen. Um die durch (8.1) gegebene Spannungsabhängigkeit der Randkonzentrationen überblicken zu können, wollen wir zwischen dem symmetrischen und dem unsymmetrischen pn-Kontakt unterscheiden. 


\section{a) Der symmetrische pn-Kontakt}

Für diesen Fall, der durch die beiden Beziehungen $Q=1, \quad\left(b_{\mathrm{n}} n_{\mathrm{D}} \varepsilon_{\mathrm{p}}\right) /\left(b_{\mathrm{p}} n_{\mathrm{A}} \varepsilon_{\mathrm{n}}\right)=1$ gekennzeichnet ist, folgt aus (8.1) :

$$
\begin{aligned}
& p_{\mathrm{R}} / n_{\mathrm{A}}=p_{\mathrm{R}}{ }^{0} / n_{\mathrm{A}}=\exp \left\{-V_{\mathrm{D}} / 2 \mathfrak{B}\right\} ; \\
& n_{\mathrm{R}} / n_{\mathrm{D}}=n_{\mathrm{R}} 0 / n_{\mathrm{D}}=\exp \left\{-V_{\mathrm{D}} / 2 \mathfrak{V}\right\} .
\end{aligned}
$$

Hier sind, wie zu erwarten, die relativen Randkonzentrationen in den beiden Halbleitern gleich groß und von der angelegten Spannung unabhängig.

\section{b) Der unsymmetrische pn-Kontakt}

Als unsymmetrischen pn-Kontakt wollen wir nur den Fall $n_{\mathrm{D}}>n_{\mathrm{A}}$ näher betrachten, da sich für $n_{\mathrm{D}}<n_{\mathrm{A}}$ nichts Neues ergibt, und sich die entsprechenden Beziehungen durch Vertauschen der Parameter des n-Leiters mit denen des p-Leiters aus den für $n_{\mathrm{D}}>n_{\mathrm{A}}$ gültigen Formeln ergeben.

Unter den Voraussetzungen

$$
Q>1, \quad\left(b_{\mathrm{n}} n_{\mathrm{D}} \varepsilon_{\mathrm{p}}\right) /\left(b_{\mathrm{p}} n_{\mathrm{A}} \varepsilon_{\mathrm{n}}\right)>1
$$

ergibt sich aus (8.1)

für das thermische Gleichgewicht bei $U=0$

$$
\begin{aligned}
& p_{\mathrm{R}}{ }^{0}=n_{\mathrm{A}} \exp \left\{-V_{\mathrm{D}} / \mathfrak{P}\left(1+Q^{-1}\right)\right\} ; \\
& n_{\mathrm{R}}{ }^{0}=n_{\mathrm{D}} \exp \left\{-V_{\mathrm{D}} / \mathfrak{P}(1+Q)\right\} .
\end{aligned}
$$

(Diese Randkonzentrationen stimmen infolge der Näherungen (7.3) nicht exakt mit denen überein, die man aus dem Nomogramm Abb. 5 erhält. Die maximale Abweichung beträgt einen Faktor $e=2,718$, was bei den aus Abb. 5 ersichtlichen Größenordnungen kaum eine Rolle spielt.)

Für nicht zu hohe Werte der Spannung $U$ folgt durch Entwickeln der Wurzel in (8.1) und einige durch die Unsymmetrie gerechtfertigte Vernachlässigungen

$$
\begin{aligned}
& p_{\mathrm{R}}=p_{\mathrm{R}}{ }^{0} \exp \{+U / \mathfrak{B}(1+Q)\} ; \\
& n_{\mathrm{R}}=n_{\mathrm{R}}{ }^{0} \exp \{-U / \mathfrak{B}(1+Q)\} .
\end{aligned}
$$

Für $U \rightarrow \infty$, d. h. für sehr hohe Sperrbelastung ergibt sich schließlich nach einigen Umformungen

$p_{\mathrm{R}}=\sqrt{b_{\mathrm{n}} \varepsilon_{\mathrm{p}} / b_{\mathrm{p}} \varepsilon_{\mathrm{n}}} \sqrt{n_{\mathrm{R}} p_{\mathrm{R}}}, n_{\mathrm{R}}=\sqrt{b_{\mathrm{p}} \varepsilon_{\mathrm{n}} / b_{\mathrm{n}} \varepsilon_{\mathrm{p}}} \sqrt{n_{\mathrm{R}} p_{\mathrm{R}}}$.

Das Ergebnis läßt sich folgendermaßen ausdrükken: Im Falle des unsymmetrischen pn-Kontaktes ist im thermischen Gleichgewicht die Randkonzentration des störstellenarmen Halbleiters extrem klein gegenüber derjenigen des stark dotierten Halbleiters. Beide Randkonzentrationen sind spannungsabhängig, die des störstellenarmen Halbleiters steigt mit zunehmender Spannung in Sperrichtung exponentiell an, die des störstellenreichen nimmt exponentiell ab. Diese Änderungen erfolgen mit zunehmender Spannung um so langsamer, je verschiedener die Störstellendichten der beiden Halbleiter sind. Für sehr hohe Sperrspannungen nehmen beide Randkonzentrationen Werte an, die nach (8.6) im wesentlichen nur von dem Verhältnis der Beweglichkeiten der Majoritätsträger abhängen. [Das in (8.6) auftretende Produkt $n_{\mathrm{R}} p_{\mathrm{R}}$ ist ja nach (6.3) unabhängig von den Störstellendichten!] Man erkennt leicht, daß diese sich für hohe Sperrspannungen einstellenden Werte dem Fall entsprechen, in dem in beiden Halbleitern an der Grenze bei $x=0$ reine Feldströme fließen. Dann gilt nämlich

$$
i_{\mathrm{n}}=e b_{\mathrm{n}} n_{\mathrm{R}} E_{\mathrm{nR}} \quad \text { und } i_{\mathrm{p}}=e b_{\mathrm{p}} p_{\mathrm{R}} E_{\mathrm{pR}},
$$

woraus mit $i_{\mathrm{n}}=i_{\mathrm{p}}$ und $\varepsilon_{\mathrm{n}} E_{\mathrm{nR}}=\varepsilon_{\mathrm{p}} E_{\mathrm{pR}}$ die Gln. (8.6) folgen.

\section{c) Der extrem unsymmetrische pn-Kontakt}

Der extrem unsymmetrische pn-Kontakt, gekennzeichnet durch $Q \rightarrow \infty$, soll hier deshalb gesondert betrachtet werden, da er rein anschaulich mit dem Metall-Halbleiter-Kontakt vergleichbar sein sollte. Für ihn ergibt sich aus (8.4) und (8.5), daß die Randkonzentrationen von der Spannung $U$ unabhängig sind und die Werte

$p_{\mathrm{R}}=p_{\mathrm{R}}{ }^{0}=n_{\mathrm{A}} \exp \left\{-V_{\mathrm{D}} / \mathfrak{B}\right\}, n_{\mathrm{R}}=n_{\mathrm{R}}^{0}=n_{\mathrm{D}}$

annehmen. Das bedeutet, da $\beta$ der extrem hochdotierte Halbleiter keine Verarmungsrandschicht mehr besitzt, und daß die Randkonzentration des schwach dotierten Halbleiters durch die Gesamtdiffusionsspannung $V_{\mathrm{D}}$ bestimmt wird. Dies entspricht in der Tat vollkommen den Verhältnissen beim MetallHalbleiter-Kontakt nach Sсноттку.

\section{Die Gleichstromkennlinie des pn-Kontaktes}

Zur Ermittlung der Gleichstromkennlinie gehen wir von Gl. (4.6) aus und erhalten daraus mit (7.5)

$$
i=e b_{\mathrm{p}} n_{\mathrm{A}} \frac{E_{\mathrm{pR}}}{a_{\mathrm{p}}} \cdot \frac{\exp \left\{U_{\mathrm{p}} / \mathfrak{B}\right\}-1}{\exp \left\{\left(V_{\mathrm{D}_{\mathrm{p}}}+U_{\mathrm{p}}\right) / \mathfrak{B}\right\}-1} .
$$

Setzt man hierin (7.3), (7.4) und (8.1) ein, so erhält man nach einer langwierigen, aber elementaren Rechnung und einigen Umformungen folgenden Ausdruck 


$$
\begin{aligned}
& i=\frac{\mathfrak{B}}{R_{0}}\left[\sqrt{\frac{b_{\mathrm{p}} n_{\mathrm{A}}}{b_{\mathrm{n}} n_{\mathrm{D}}}} \varepsilon_{\mathrm{n}} a_{\mathrm{n}}{ }^{0} \cdot\left(\exp \left\{\frac{V_{\mathrm{D}}}{\mathfrak{B}(1+Q)}\right\}-1\right)+\sqrt{\frac{b_{\mathrm{n}} n_{\mathrm{D}}}{b_{\mathrm{p}} n_{\mathrm{A}}}} \varepsilon_{\mathrm{p}} a_{\mathrm{p}}{ }^{0} \cdot\left(\exp \left\{\frac{V_{\mathrm{D}}}{\mathfrak{B}\left(1+Q^{-1}\right)}\right\}-1\right)\right] \sqrt{1+\frac{U}{V_{\mathrm{D}}}} \\
& \cdot\left\{\left[\frac{1}{4}\left\{\frac{\sqrt{b_{\mathrm{p}} n_{\mathrm{A}} / b_{\mathrm{n}} n_{\mathrm{D}}}}{\varepsilon_{\mathrm{p}} a_{\mathrm{p}}\left(\exp \left\{\left(V_{\mathrm{D}}+U\right) / \mathfrak{B}\left(1+Q^{-1}\right)\right\}-1\right)}+\frac{\sqrt{b_{\mathrm{n}} n_{\mathrm{D}} / b_{\mathrm{p}} n_{\mathrm{A}}}}{\varepsilon_{\mathrm{n}} a_{\mathrm{n}}\left(\exp \left\{\left(V_{\mathrm{D}}+U\right): \mathcal{B}(1+Q)\right\}-1\right)}\right\}^{2}\right.\right. \\
& \left.+\frac{\exp \{U / \mathfrak{B}\}-1}{\varepsilon_{\mathrm{n}} a_{\mathrm{n}}\left(\exp \left\{\left(V_{\mathrm{D}}+U\right) / \mathfrak{B}(1+Q)\right\}-1\right) \cdot \varepsilon_{\mathrm{p}} a_{\mathrm{p}}\left(\exp \left\{\left(V_{\mathrm{D}}+U\right) / \mathfrak{B}\left(1+Q^{-1}\right)\right\}\right)-1}\right]^{1 / 2} \\
& \left.-\frac{1}{2}\left[\frac{\sqrt{b_{\mathrm{p}} n_{\mathrm{A}} / b_{\mathrm{n}} n_{\mathrm{D}}}}{\varepsilon_{\mathrm{p}} a_{\mathrm{p}}\left(\exp \left\{\left(V_{\mathrm{D}}+U\right) / \mathfrak{B}\left(1+Q^{-1}\right)\right\}-1\right)}+\frac{\sqrt{b_{\mathrm{n}} n_{\mathrm{D}} / b_{\mathrm{p}} n_{\mathrm{A}}}}{\varepsilon_{\mathrm{n}} a_{\mathrm{n}}\left(\exp \left\{\left(V_{\mathrm{D}}+U\right) / \mathfrak{B}(1+Q)\right\}-1\right)}\right]\right\}
\end{aligned}
$$

mit dem Nullwiderstand $R_{0}$ :

$$
R_{0}=\mathfrak{V} \sqrt{\frac{\varepsilon_{\mathrm{V}}}{8 \pi e V_{\mathrm{D}}}} \sqrt{\frac{1}{\varepsilon_{\mathrm{n}} n_{\mathrm{D}}}+\frac{1}{\varepsilon_{\mathrm{p}} n_{\mathrm{A}}}}\left\{\frac{\varepsilon_{\mathrm{n}} a_{\mathrm{n}}{ }^{0}\left(\exp \left\{V_{\mathrm{D}} / \mathfrak{B}(1+Q)\right\}-1\right)}{e b_{\mathrm{n}} n_{\mathrm{D}}}+\frac{\varepsilon_{\mathrm{p}} a_{\mathrm{p}}{ }^{0}\left(\exp \left\{V_{\mathrm{D}} / \mathfrak{B}\left(1+Q^{-1}\right)\right\}-1\right)}{e b_{\mathrm{p}} n_{\mathrm{A}}}\right\},
$$

$a_{\mathrm{n}}^{0}$ und $a_{\mathrm{p}}^{0}$ sind die Werte der Zahlenfaktoren $a_{\mathrm{n}}$ und $a_{\mathrm{p}}$ für $U=0$.

Gl. (9.2) stellt die geschlossene Darstellung der Gleichstromkennlinie eines pn-Kontaktes dar. Sie ist völlig symmetrisch bezüglich der Parameter der beiden Halbleiter. Dieser recht unübersichtliche, allgemeine Ausdruck vereinfacht sich für verschiedene Bereiche der Parameter ganz wesentlich und wir unterscheiden wieder zwischen dem symmetrischen und dem unsymmetrischen pn-Kontakt.

\section{a) Gleichstromkennlinie des symmetrischen pn-Kontaktes}

Für den hier durch

$$
Q=1, \quad\left(b_{\mathrm{n}} n_{\mathrm{D}} \varepsilon_{\mathrm{p}}\right) /\left(b_{\mathrm{p}} n_{\mathrm{A}} \varepsilon_{\mathrm{n}}\right)=1
$$

gekennzeichneten Fall ergibt sich aus (9.2) als Kennliniengleichung für den gesamten Spannungsbereich, wie nach Abschn. 3 zu erwarten,

$$
i=2 \frac{\mathfrak{B}}{R_{0}} \sqrt{1+\frac{U}{V_{\mathrm{D}}}}\left(1-\exp \left\{-\frac{U}{2 \mathfrak{V}}\right\}\right)
$$

mit $\quad R_{0}=\mathfrak{V} \sqrt{\frac{\varepsilon_{\mathrm{p}} \varepsilon_{\mathrm{V}}}{\pi e n_{\mathrm{A}} V_{\mathrm{D}}}} \frac{1}{e b_{\mathrm{p}} n_{\mathrm{A}}} \exp \left\{\frac{V_{\mathrm{D}}}{2 \mathfrak{B}}\right\}$.

Diese Kennliniengleichung entspricht derjenigen des Metall-Halbleiter-Kontaktes nach Sсноттку (s. Abschnitt 3) bis auf den Exponenten der $e$-Funktion, der hier $U / 2 \mathfrak{V}$ heißt an Stelle von $U / \mathfrak{V}$. Das hat zur Folge, daß der Durchlaßstrom $(U<0)$ mit $\exp \{U / 2 \mathfrak{B}\}$ ansteigt und nicht mit $\exp \{U / \mathfrak{B}\}$ (s. Abb. 6 bis 8 ).

\section{b) Gleichstromkennlinie des unsymmetrischen pn-Kontaktes}

Wir betrachten hier wiederum nur den Fall $n_{\mathrm{D}}>n_{\mathrm{A}}$ bzw. $b_{\mathrm{n}}>b_{\mathrm{p}}$, da sich die Kennliniengleichung für

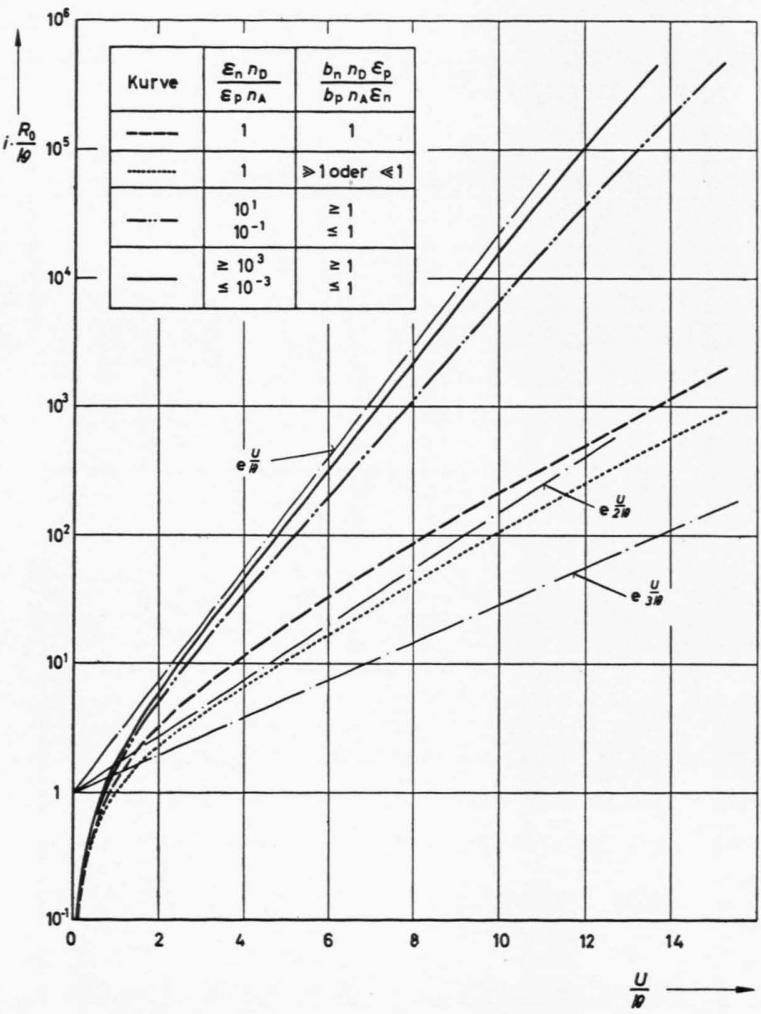

Abb. 6. Durchlaßkennlinie des pn-Kontaktes $\left(V_{\mathrm{D}}=20 \mathfrak{V}\right)$.

$n_{\mathrm{D}}<n_{\mathrm{A}}$ bzw. $b_{\mathrm{n}}<b_{\mathrm{p}}$ wieder durch Vertauschen der Parameter der beiden Halbleiter ergibt. Man unterscheidet zweckmäßig zwischen einem Bereich hoher Sperrspannungen, in dem unter der Wurzel in (9.2) der zweite Term groß gegen den ersten ist, und dem übrigen Bereich nicht zu hoher Spannungen in Sperrrichtung und Durchlaßrichtung, in dem der erste Term unter der Wurzel in (9.2) groß gegen den zweiten ist. 


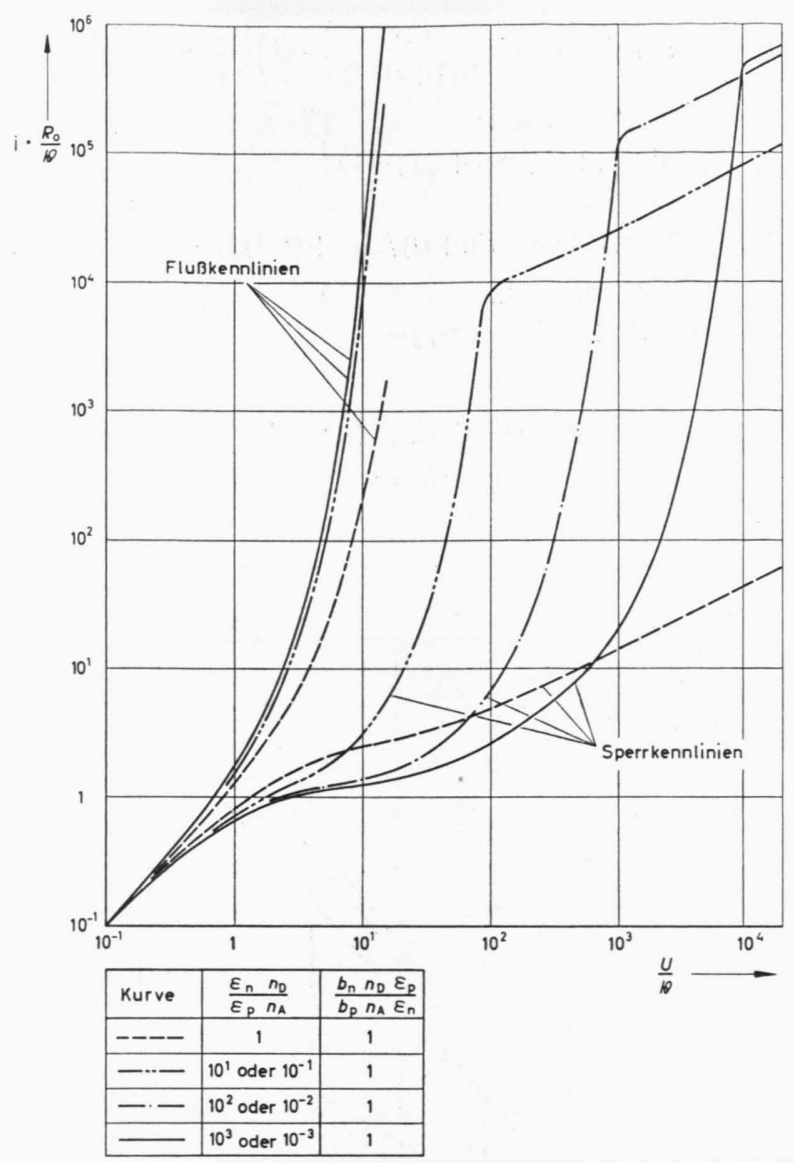

Abb. 7. Gleichstromkennlinie des pn-Kontaktes $\left(V_{\mathrm{D}}=20 \mathfrak{Z}\right)$.

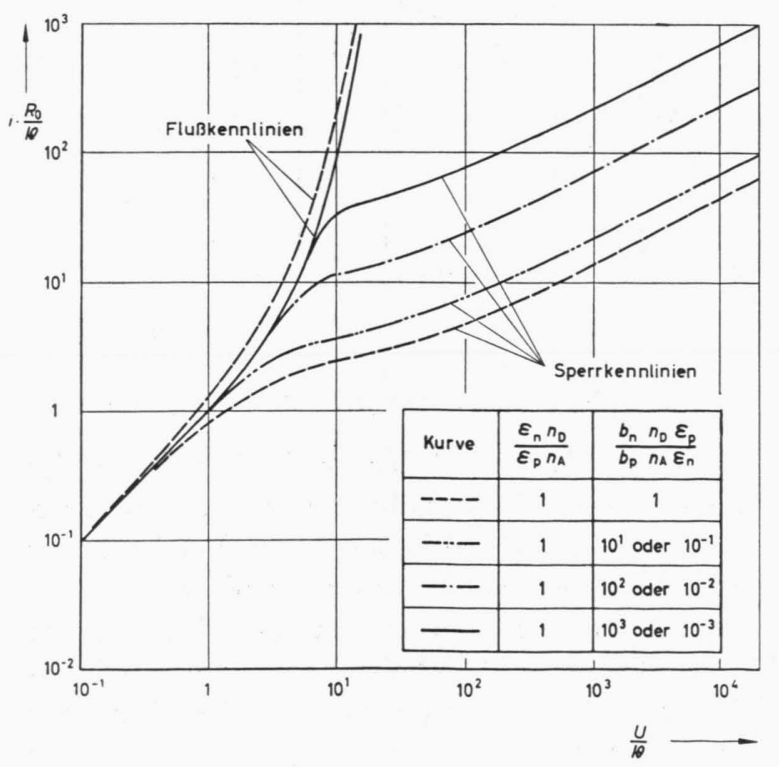

Abb. 8. Gleichstromkennlinie des pn-Kontaktes $\left(V_{\mathrm{D}}=20 \mathfrak{Z}\right)$.
Man erhält dann durch Entwickeln der Wurzel in (9.2) für die beiden Fälle

$$
Q=1, \quad b_{\mathrm{n}} n_{\mathrm{D}} \varepsilon_{\mathrm{p}} / b_{\mathrm{p}} n_{\mathrm{A}} \varepsilon_{\mathrm{n}} \gg 1
$$

und $\quad Q>1, \quad b_{\mathrm{n}} n_{\mathrm{D}} / b_{\mathrm{p}} n_{\mathrm{A}}>1$

dieselben Kennliniengleichungen, und zwar für den Bereich nicht zu hoher Spannungen

$i=\frac{\mathfrak{V}}{R_{0}} \sqrt{1+\frac{U}{V_{\mathrm{D}}}} \exp \left\{\frac{U}{\mathfrak{B}(1+Q)}\right\}\left(1-\exp \left\{-\frac{U}{\mathfrak{V}}\right\}\right)$

und für sehr hohe Sperrspannungen

$i=\frac{\mathfrak{V}}{R_{0}} \sqrt{\frac{b_{\mathrm{n}} n_{\mathrm{D}} \varepsilon_{\mathrm{p}}}{b_{\mathrm{p}} n_{\mathrm{A}} \varepsilon_{\mathrm{n}}}} \exp \left\{\frac{V_{\mathrm{D}}}{2 \mathfrak{V}\left(1-Q^{-1}\right) /\left(1+Q^{-1}\right)}\right\}$.

Der Übergang zwischen den beiden Spannungsbereichen liegt für $Q=1, \quad\left(b_{\mathrm{n}} n_{\mathrm{D}} \varepsilon_{\mathrm{p}}\right) /\left(b_{\mathrm{p}} n_{\mathrm{A}} \varepsilon_{\mathrm{n}}\right) \gg 1$ bei $U / \mathfrak{B}=\ln \left\{\frac{1}{4}\left(b_{\mathrm{n}} n_{\mathrm{D}} \varepsilon_{\mathrm{p}}\right) /\left(b_{\mathrm{p}} n_{\mathrm{A}} \varepsilon_{\mathrm{n}}\right)\right\}$ und für $Q>1$, $\left(b_{\mathrm{n}} n_{\mathrm{D}}\right) /\left(b_{\mathrm{p}} n_{\mathrm{A}}\right)>1$ bei $U \approx Q V_{\mathrm{D}} / 2$.

Für den Nullwiderstand ergibt sich

$R_{0}=\mathfrak{V} \sqrt{\frac{\varepsilon_{\mathrm{V}}}{8 \pi e V_{\mathrm{D}}}} \sqrt{\frac{1}{\varepsilon_{\mathrm{n}} n_{\mathrm{D}}}+\frac{1}{\varepsilon_{\mathrm{p}} n_{\mathrm{A}}}} \cdot \frac{\varepsilon_{\mathrm{p}}}{e b_{\mathrm{p}} p_{\mathrm{R}}^{0}}$.

In Abb. 6 bis 8 sind die Kennlinien für einige Fälle nach den Gln. (9.6) und (9.7) dargestellt, wobei ein Bahnwiderstand - auch in Durchlaßrichtung nicht berücksichtigt wurde.

Die wesentlichen Merkmale der Gleichstromkennlinie des unsymmetrischen pn-Kontaktes lassen sich hiernach folgendermaßen beschreiben:

Der Anstieg der Flußkennlinie liegt je nach dem Wert des Verhältnisses $Q=\varepsilon_{\mathrm{n}} n_{\mathrm{D}} / \varepsilon_{\mathrm{p}} n_{\mathrm{A}}$ zwischen $\exp \{U / \mathfrak{B}\}$ und $\exp \{U / 2 \mathfrak{B}\}$. Der Anstieg $\exp \{U / \mathfrak{V}\}$ wird bei starker Unsymmetrie in den Störstellendichten erreicht und entspricht der Tatsache, daß dann praktisch die gesamte von außen angelegte Spannung $U$ über der Raumladungsschicht des störstellenarmen Halbleiters abfällt. Der Anstieg mit $\exp \{U / 2 \mathfrak{B}\}$ tritt bei $\varepsilon_{\mathrm{n}} n_{\mathrm{D}}=\varepsilon_{\mathrm{p}} n_{\mathrm{A}}$ auf, da hier die Spannung $U$ je zur Hälfte über den beiden Raumladungsschichten abfällt [s. Gl. (7.3) ].

Die Sperrkennlinie zeigt im allgemeinen keinen Sättigungscharakter, sondern steigt exponentiell mit der Spannung an, und zwar um so langsamer, je mehr sich die Störstellendichten der beiden Halbleiter unterscheiden. Dies ist der charakteristische und wesentliche Unterschied zwischen der Sperrkennlinie eines pn-Kontaktes und derjenigen eines Metall-Halbleiter-Kontaktes. Die Ursache hierfür ist 
die in Abschn. 8 betrachtete Änderung der Randkonzentrationen $n_{\mathrm{R}}$ und $p_{\mathrm{R}}$ mit der Spannung $U$. Erst für Spannungen, bei denen an der Grenzschicht zwischen den beiden Halbleitern nur reine Feldströme fließen, bei denen die Randkonzentrationen also ihre durch (8.6) gegebenen Grenzwerte angenommen haben, nimmt der Sperrstrom nur mit $U^{1 / 2}$ zu (s. Abb. 7).

Bemerkenswert ist noch, daß in dem Spezialfall $\varepsilon_{\mathrm{n}} n_{\mathrm{D}}=\varepsilon_{\mathrm{p}} n_{\mathrm{A}}$ bei stark verschiedenen Beweglichkeiten der Majoritätsträger auch der Sperrstrom zunächst stärker als proportional $U$ zunimmt, um erst bei höheren Spannungen einem $U^{1 / 2}$-Gesetz zu folgen (s. Abb. 8).

\section{c) Die Gleichstromkennlinie des extrem unsymmetrischen pn-Kontaktes}

Für den extrem unsymmetrischen pn-Kontakt, gekennzeichnet durch $Q \rightarrow \infty$, ergibt sich aus (9.6) die Kennliniengleichung:

$$
i=\frac{\mathfrak{B}}{R_{0}} \sqrt{1+\frac{U}{V_{\mathrm{D}}}} \cdot(1-\exp \{-U / \mathfrak{V}\}) .
$$

Diese Beziehung stimmt vollkommen überein mit der von Sсноттку angegebenen Gleichstromkennlinie des Metall-Halbleiter-Kontaktes.

\section{Der Widerstand $R=U / i$ und der differentielle Widerstand $R_{\text {diff }}=\mathrm{dU} / \mathrm{d} i$}

Besonders geeignet für einen Vergleich der vorliegenden Theorie mit Messungen an pn-Kontakten ist neben der Gleichstromkennlinie selbst die Spannungsabhängigkeit des Widerstandes $R=U / i$ und des differentiellen Widerstandes $R_{\text {diff }}=\mathrm{d} U / \mathrm{d} i$. Man gewinnt diese Beziehungen leicht aus den Kennliniengleichungen (9.4), (9.6) und (9.9). Abb. 9 zeigt das Ergebnis für einige Werte des Verhältnisses $Q=\varepsilon_{\mathrm{n}} n_{\mathrm{D}} / \varepsilon_{\mathrm{p}} n_{\mathrm{A}}$. Es zeigt sich, daß die Sperrwiderstände des symmetrischen $(Q=1)$ und des extrem unsymmetrischen $(Q \rightarrow \infty)$ pn-Kontaktes mit der Sperrspannung kontinuierlich zunehmen. Für den unsymmetrischen pn-Kontakt $(Q \neq 1)$ ergibt sich jedoch als charakteristischer Verlauf zunächst ein Anstieg bis zu einem Maximum und ein anschließender steiler Abfall, der im wesentlichen exponentiell verläuft. Die Spannung, bei der das Maximum auftritt, und die Höhe des Maximums hängen dabei sehr stark von dem Verhältnis $Q$ ab. Für noch höhere Spannungen

$$
U>\frac{1}{2} V_{\mathrm{D}} Q \text { für } Q>1
$$

bzw. $\quad U>\frac{1}{2} V_{\mathrm{D}} / Q$ für $Q<1$

nimmt der Sperrwiderstand wieder zu mit $U^{1 / 2}$.

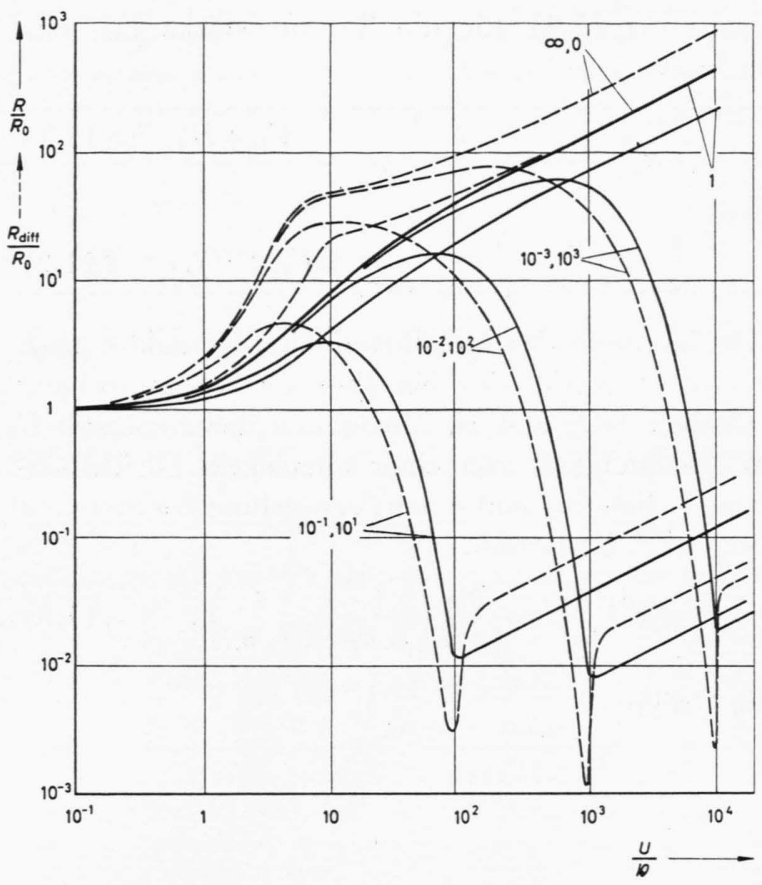

Abb. 9. Sperrwiderstand des pn-Kontaktes; $R=U / i$ und $R_{\text {diff }}=\mathrm{d} U / \mathrm{d} i$ in Abhängigkeit von der in Sperrichtung angelegten Spannung $U$. [ $\left.V_{\mathrm{D}}=20 \mathfrak{V},\left(b_{\mathrm{n}} n_{\mathrm{D}} \varepsilon_{\mathrm{p}}\right) /\left(b_{\mathrm{p}} n_{\mathrm{A}} \varepsilon_{\mathrm{n}}\right)=1\right]$, Parameter $Q=\left(\varepsilon_{\mathrm{n}} n_{\mathrm{D}}\right) /\left(\varepsilon_{\mathrm{p}} n_{\mathrm{A}}\right)$.

\section{Die Kapazität des pn-Kontaktes}

Da die an der Grenze der beiden Halbleiter auftretenden Raumladungsschichten durch eine von außen angelegte Spannung $U$ sowohl in ihrer Ausdehnung als auch hinsichtlich der Raumladungsdichte beeinflußt werden, verhält sich der pn-Kontakt bei Anliegen einer Wechselspannung wie ein Kondensator. Sind $Q^{*}$ die gesamte Raumladung eines Vorzeichens pro Flächeneinheit und $\varrho_{\mathrm{n}}=e\left(n_{\mathrm{D}}-n\right)$ die Raumladungsdichte im n-Leiter, so ist die Kapazität $C$ pro Flächeneinheit

$$
C=\frac{\mathrm{d} Q^{*}}{\mathrm{~d} U}=\frac{\mathrm{d}}{\mathrm{d} U} \int_{-l_{\mathrm{n}}}^{0} e\left(n_{\mathrm{D}}-n\right) \mathrm{d} x,
$$


und weiter nach der Porsson-Gleichung (3.2)

$$
C=\frac{\mathrm{d}}{\mathrm{d} U} \frac{\varepsilon_{\mathrm{n}} \varepsilon_{\mathrm{V}}}{4 \pi} \int_{-l_{\mathrm{n}}}^{0} \frac{\mathrm{d} E_{\mathrm{n}}(x) \mathrm{d} x}{\mathrm{~d} x}=\frac{\varepsilon_{\mathrm{V}}}{4 \pi} \frac{\mathrm{d}\left(\varepsilon_{\mathrm{n}} E_{\mathrm{nR}}\right)}{\mathrm{d} U},
$$

wobei wir die Bahnfeldstärke bei $x=-l_{\mathrm{n}}$ wiederum gegenüber der Randfeldstärke bei $x=0$ vernachlässigen. Setzt man hier die in Abschn. 7 gewonnene Beziehung (7.4) für die Randfeldstärke $E_{\mathrm{nR}}$ ein, so folgt

$C=\left(\frac{e \varepsilon_{\mathrm{v}}}{8 \pi}\right)^{1 / 2}\left(\frac{1}{\varepsilon_{\mathrm{n}} n_{\mathrm{D}}}+\frac{1}{\varepsilon_{\mathrm{p}} n_{\mathrm{A}}}\right)^{-1 / 2}\left(V_{\mathrm{D}}+U\right)^{-1 / 2}$

und

$$
\frac{1}{C^{2}}=\frac{8 \pi}{e \varepsilon_{\mathrm{v}}}\left(\frac{1}{\varepsilon_{\mathrm{n}} n_{\mathrm{D}}}+\frac{1}{\varepsilon_{\mathrm{p}} n_{\mathrm{A}}}\right)\left(V_{\mathrm{D}}+U\right) .
$$

Wie im Falle des Metall-Halbleiter-Kontaktes nach Sсноттку ergibt sich ein linearer Zusammenhang zwischen $1 / C^{2}$ und $U$. Messungen der Kapazität $C$ in Abhängigkeit von einer angelegten Gleichspannung $U$ liefern somit durch Auswertung der Steigung $\operatorname{der}\left(1 / C^{2}, U\right)$-Geraden,

$$
\frac{\mathrm{d}\left(1 / C^{2}\right)}{\mathrm{d} U}=\frac{8 \pi}{e \varepsilon_{\mathrm{V}}}\left(\frac{1}{\varepsilon_{\mathrm{n}} n_{\mathrm{D}}}+\frac{1}{\varepsilon_{\mathrm{p}} n_{\mathrm{A}}}\right),
$$

die Größe $\left(\frac{1}{\varepsilon_{\mathrm{n}} n_{\mathrm{D}}}+\frac{1}{\varepsilon_{\mathrm{p}} n_{\mathrm{A}}}\right)$.

\section{Schlußbemerkungen}

Die vorstehenden Überlegungen zeigen, daß das Gleichstromverhalten eines pn-Kontaktes sehr stark von dem Verhältnis der Störstellenkonzentrationen in den beiden Halbleitern abhängt. Für den symmetrischen pn-Kontakt ergibt sich die Gleichstromkennlinie zweier hintereinander geschalteter MetallHalbleiter-Kontakte. Im Falle des unsymmetrischen pn-Kontaktes werden die Randkonzentrationen der Majoritätsträger an der Grenze der beiden Halbleiter abhängig von der von außen angelegten Spannung. Das hat einen im wesentlichen exponentiellen Anstieg des Sperrstromes in einem von dem Verhältnis der Störstellendichten abhängigen Spannungsbereich und das Auftreten eines Maximums des Sperrwiderstandes zur Folge.

Über die Anwendung der Theorie auf den Selengleichrichter und ihren Vergleich mit Experimenten wird in einer weiteren Arbeit berichtet werden, wobei auch die in den hier angegebenen Beziehungen enthaltenen Temperaturabhängigkeiten näher betrachtet werden sollen.

Für anregende Diskussionen bin ich Herrn Dr. H. Lauckner und Herrn H.-P. Hempel zu großem Dank verpflichtet. 